\title{
Spatial Variability of Middle East Respiratory Syndrome Coronavirus Survival Rates and Mortality Hazard in Saudi Arabia, 2012-2019
}

\author{
Khalid Hussain Al-Ahmadi ${ }^{1}$, Mohammed Hussain Alahmadi ${ }^{\text {Corresp., } 1}{ }^{\text {, Ali Saeed Al-Zahrani }}{ }^{2}$, Maged Gomaa Hemida \\ Corresp. 3, 4 \\ ${ }^{1}$ King Abdulaziz City for Science and Technology, Riyadh, Riyadh, Saudi Arabia \\ 2 King Faisal Specialist Hospital and Research Centre, Riyadh, Riyadh, Saudi Arabia \\ 3 Department of Microbiology, College of Veterinary Medicine, King Faisal University, Al-Hufuf, Al-Hasa, Saudi Arabia \\ 4 Department of Virology, faculty of veterinary medicine, Kafrelsheikh University, Kafrelsheikh, Kafrelsheikh, Egypt \\ Corresponding Authors: Mohammed Hussain Alahmadi, Maged Gomaa Hemida \\ Email address: mhalahmadi@kacst.edu.sa, mhemida@kfu.edu.sa
}

About $83 \%$ of laboratory-confirmed Middle East respiratory syndrome coronavirus (MERSCoV) cases have emerged from Saudi Arabia, which has the highest overall mortality rate worldwide. This retrospective study assesses the impact of spatial/patient characteristics for 14- and 45-day MERS-CoV mortality using 2012-2019 data reported across Saudi regions and provinces. The Kaplan-Meier estimator was employed to estimate MERS-CoV survival rates, Cox proportional-hazards $(\mathrm{CPH})$ models were applied to estimate hazard ratios (HRs) for 14- and 45-day mortality predictors, and univariate local spatial autocorrelation and multivariate spatial clustering analyses were used to assess the spatial correlation. The 14-day, 45-day, and overall mortality rates (with estimated survival rates) were $25.52 \%$ (70.20\%), 32.35\% (57.70\%), and $37.30 \%$ (56.50\%), respectively, with no significant rate variations between Saudi regions and provinces. Nationally, the $\mathrm{CPH}$ multivariate model identified that being elderly (age $\geq 61$ ), being a non-healthcare worker (non-HCW), and having an underlying comorbidity were significantly related to 14-day mortality ( $H R=2.10,10.12$, and 4.11 , respectively; $p<0.0001)$. The 45-day mortality model identified similar risk factors but with an additional factor: patients aged 41-60 (HR $=1.44 ; \mathrm{p}<0.0001)$. Risk factors similar to those in the national model were observed in the Central, East, and West regions and Riyadh, Makkah, Eastern, Madinah, and Qassim provinces but with varying HRs. Spatial clusters of MERS-CoV mortality in the provinces were identified based on the risk factors $\left(r^{2}=0.85-0.97\right)$ : Riyadh (Cluster 1$)$, Eastern, Makkah, and Qassim (Cluster 2), and other provinces in the north and south of the country (Cluster 3). The estimated HRs for the 14- and 45-day mortality varied spatially by province. For 45-day mortality, the highest HRs were found in Makkah (age $\geq 61$ and non- 
HCWs), Riyadh (comorbidity), and Madinah (age 41-60). Coming from Makkah (HR $=1.30$ and 1.27) or Qassim province ( $H R=1.77$ and 1.70) was independently related to higher 14- and 45-day mortality, respectively. MERS-CoV patient survival could be improved by implementing appropriate interventions for the elderly, those with comorbidities, and nonHCW patients. 
1 Spatial Variability of Middle East Respiratory Syndrome Coronavirus Survival Rates and

2 Mortality Hazard in Saudi Arabia, 2012-2019

3 Khalid Hussain Al-Ahmadi ${ }^{1}$, Mohammed Hussain Alahmadi ${ }^{1}$, Ali Saeed Al-Zahrani ${ }^{2}$, and

4 Maged Gomaa Hemida ${ }^{3,4}$

$5 \quad 1$ King Abdulaziz City for Science and Technology, Riyadh, Riyadh, Saudi Arabia

$6 \quad 2$ King Faisal Specialist Hospital and Research Centre, Riyadh, Riyadh, Saudi Arabia

73 Department of Microbiology, College of Veterinary Medicine, King Faisal University, Al

8 Hofuf, Al-Ahsa, Saudi Arabia

$9{ }^{4}$ Department of Virology, Faculty of Veterinary Medicine, Kafrelsheikh University, Egypt

10

11 Correspondence Authors:

$12{ }^{1}$ Mohammed Alahmadi

13 P.O. Box 6086, Riyadh 11442, Saudi Arabia.

14 Email Address: mhalahmadi@kacst.edu.sa

$15 \quad 3,4$ Maged Hemida

16 Department of Microbiology, College of Veterinary Medicine, King Faisal University, Al

17 Hofuf, Al-Ahsa, Saudi ArabiaEmail Address: mhemida@kfu.edu.sa 


\section{Abstract:}

About $83 \%$ of laboratory-confirmed Middle East respiratory syndrome coronavirus (MERS$\mathrm{CoV}$ ) cases have emerged from Saudi Arabia, which has the highest overall mortality rate worldwide. This retrospective study assesses the impact of spatial/patient characteristics for 14and 45-day MERS-CoV mortality using 2012-2019 data reported across Saudi regions and provinces. The Kaplan-Meier estimator was employed to estimate MERS-CoV survival rates, Cox proportional-hazards $(\mathrm{CPH})$ models were applied to estimate hazard ratios (HRs) for 14and 45-day mortality predictors, and univariate local spatial autocorrelation and multivariate spatial clustering analyses were used to assess the spatial correlation. The 14-day, 45-day, and overall mortality rates (with estimated survival rates) were $25.52 \%(70.20 \%), 32.35 \%$ $(57.70 \%)$, and $37.30 \%(56.50 \%)$, respectively, with no significant rate variations between Saudi regions and provinces. Nationally, the $\mathrm{CPH}$ multivariate model identified that being elderly (age $\geq 61$ ), being a non-healthcare worker (non-HCW), and having an underlying comorbidity were significantly related to 14-day mortality $(\mathrm{HR}=2.10,10.12$, and 4.11, respectively; $\mathrm{p}<0.0001)$. The 45-day mortality model identified similar risk factors but with an additional factor: patients aged $41-60(\mathrm{HR}=1.44 ; \mathrm{p}<0.0001)$. Risk factors similar to those in the national model were observed in the Central, East, and West regions and Riyadh, Makkah, Eastern, Madinah, and Qassim provinces but with varying HRs. Spatial clusters of MERS-CoV mortality in the provinces were identified based on the risk factors $\left(\mathrm{r}^{2}=0.85-0.97\right)$ : Riyadh (Cluster 1), Eastern, Makkah, and Qassim (Cluster 2), and other provinces in the north and south of the country (Cluster 3). The estimated HRs for the 14- and 45-day mortality varied spatially by province. For 45-day mortality, the highest HRs were found in Makkah (age $\geq 61$ and non-HCWs), Riyadh (comorbidity), and Madinah (age 41-60). Coming from Makkah (HR $=1.30$ and 1.27) or Qassim province $(\mathrm{HR}=1.77$ and 1.70$)$ was independently related to higher 14- and 45-day mortality, respectively. MERS-CoV patient survival could be improved by 53 implementing appropriate interventions for the elderly, those with comorbidities, and non-HCW patients. 


\section{Introduction}

Middle East respiratory syndrome coronavirus (MERS-CoV), first reported in Saudi Arabia in 2012 (Zaki et al. 2012), is an infectious viral respiratory disease with high mortality and morbidity in humans. From 2012 through April 30, 2019, 27 countries reported a total of 2,428 MERS-CoV laboratory-confirmed cases to WHO, of which $83 \%$ were reported by Saudi Arabia (WHO 2019). The largest cluster outside the Arabian Peninsula was reported from South Korea in 2015 (Chen et al. 2017). MERS-CoV mortality rates vary by region: $34.5 \%-45 \%$ worldwide (Ahmed 2018; Chen et al. 2017; Penttinen et al. 2013; WHO 2019), 10\%-65\% in Saudi Arabia (Ahmed 2017b; Al Ghamdi et al. 2016; Alqahtani et al. 2018; Assiri et al. 2016; Assiri et al. 2013a; Coleman et al. 2017; El Bushra et al. 2017; Feikin et al. 2015; Lessler et al. 2016; Mohd et al. 2016a; Noorwali et al. 2015; Oboho et al. 2015; Prevention 2015; Saad et al. 2014; Sherbini et al. 2017), and 19.9\%-63.6\% in South Korea (Kim et al. 2019; Mizumoto et al. 2015; Nam et al. 2017; Prevention 2015). The highest MERS-CoV mortality rates reported for Saudi Arabia (Assiri et al. 2013a) and South Korea (Nam et al. 2017) were linked to nosocomial outbreaks. Some studies have estimated mortality rates between $26.6 \%$ and $59.4 \%$ (Breban et al. 2013; Liu et al. 2015; Sha et al. 2017) using data from multiple regions, including the Middle East, South Korea, and China. Due to its high mortality rate and a lack of effective antiviral treatment, preventive vaccine, or prophylactic therapy, MERS-CoV remains a public health risk (Mohd et al. 2016b).

MERS-CoV is an emergent zoonotic virus, and camel-to-human transmission is linked to direct or indirect exposure to infected dromedary camels (Camelus dromedarius) and their products (Azhar et al. 2014). Although dromedary camels have been recognized as intermediate hosts of MERS-CoV, human-to-human virus transmission has been identified as nosocomial transmission in healthcare facilities among patients and healthcare workers (HCWs) (Hui et al. 2018). It has also been identified within Saudi households (Drosten et al. 2014), raising concerns about an epidemic risk. The risk assessment of MERS-CoV mortality is crucial to improving clinical patient outcomes by implementing measures and strategies that improve patients' chances of survival and provide effective early treatment.

Primary factors related to MERS-CoV mortality are old age (Ahmed 2017b; Ahmed 2018; Alqahtani et al. 2018; Chen et al. 2017; Majumder et al. 2015) and underlying comorbidities (Ahmed 2017b; Ahmed 2018; Alqahtani et al. 2018; Assiri et al. 2013a; Majumder et al. 2015; 
86 Nam et al. 2017; Sha et al. 2017; Sherbini et al. 2017), including diabetes (Alqahtani et al. 2018;

87 Assiri et al. 2013b; Sherbini et al. 2017), cardiac diseases (Alqahtani et al. 2018; Assiri et al. 88 2013b), chronic renal disease (Alqahtani et al. 2018; Assiri et al. 2013b), kidney failure (Sherbini 89 et al. 2017), and respiratory disease (Alqahtani et al. 2018; Nam et al. 2017). The severity of the 90 illness for patients receiving vasopressor therapy (Almekhlafi et al. 2016), requiring invasive 91 mechanical ventilation (Almekhlafi et al. 2016), and with higher chest radiographic scores (Das 92 et al. 2015) is also associated with mortality risk resulting from MERS-CoV (Adegboye et al. 93 2017; Ahmed 2017a). Other symptoms associated with MERS-CoV deaths include demonstrated 94 gastrointestinal symptoms (Sherbini et al. 2017), leucocytosis (Nam et al. 2017), respiratory 95 symptoms, abnormal renal function (Nam et al. 2017), and lower blood pressures (Sherbini et al. 96 2017). Some researchers have found that being an HCW is associated with higher mortality rates 97 (Alsahafi \& Cheng 2016), but, conversely, other studies have linked being a non-healthcare 98 worker (non-HCW) with higher mortality rates (Adegboye et al. 2017; Ahmed 2017b; Ahmed 2018; Rivers et al. 2016; Sha et al. 2017). Another study found that exposure to camels and nosocomial and unknown infection sources demonstrated a higher mortality risk compared to exposure to household infection (Ahmed 2018). However, another study reported no significant association between MERS-CoV survival and exposure to camels (Sha et al. 2017). Mortality risk factors also include a longer period from onset to laboratory confirmation (Sha et al. 2017), a longer time from onset to hospitalization (Sha et al. 2017), a longer duration of symptoms (Sherbini et al. 2017), and a shorter incubation period (Cowling et al. 2015). In the Middle East, extended times from the onset of clinical signs to laboratory confirmation (4-8 days) have been reported compared to South Korea (4-5 days) (Sha et al. 2017). Saudi Arabia has reported a shorter incubation period (5.2 days) compared to South Korea (6 days) (Assiri et al. 2013a; Sha et al. 2017). The risk of mortality for MERS-CoV patients in Saudi Arabia was 4.1 times higher after 14 days and 5 times higher after 45 days compared to South Korea and other countries 111 (Ahmed 2018).

112 Previous studies have provided useful information on the mortality risk for MERS-CoV 113 patients. These studies exemplify particular patient clusters, such as critical care units 114 (Almekhlafi et al. 2016), patients in a single hospital (Saad et al. 2014), area-specific groups 115 within Saudi Arabia (Assiri et al. 2013a; Noorwali et al. 2015; Oboho et al. 2015; Sherbini et al. 116 2017), and outbreak-specific groups in light of the South Korean outbreak (Cowling et al. 2015; 
117 Kim et al. 2019; Majumder et al. 2015; Mizumoto et al. 2015). Viral and host factors, such as the

118 virus's replication and mutation rates, and local medical expertise also affect MERS-CoV

119 mortality rates (Feikin et al. 2015). Thus, mortality risk factors vary according to regional

120 characteristics, such as the strategies and measures implemented by healthcare systems,

121 hospitalization practices, and accessibility to healthcare centers (Ahmed 2018; Feikin et al.

122 2015). MERS-CoV mortality risk factors may vary geographically (Banik et al. 2016).

123 Significant spatiotemporal variations and clusters of MERS-CoV incidence have been identified

124 in Saudi Arabia (Al-Ahmadi et al. 2019). A spatial modeling approach (Adegboye et al. 2017)

125 used a geoadditive regression model to examine the risk of mortality from MERS-CoV in the

126 Arabian Peninsula. However, this study did not consider risk factors by province. Using publicly

127 available national MERS-CoV data for Saudi Arabia, (Banik et al. 2016)) assessed risk factors

128 related to severity and mortality using a relatively large dataset; however, this study lacked

129 multivariate risk modeling. One study (Ahmed 2017b) applied multivariate analysis to estimate

130 the survival rate of MERS-CoV mortality in Saudi Arabia; however, it did not examine risk

131 factors by region or province.

132 To the authors' knowledge, the risk factors for MERS-CoV mortality at the regional level in

133 Saudi Arabia have not been examined, including variations between mortality risk factors in

134 different geographical areas (both regions and provinces). It is not evident whether the mortality

135 risk factors for MERS-CoV vary by region in Saudi Arabia. It is essential to perform multi-

136 region and multi-province recognition to compare MERS-CoV patients in relation to their

137 clinical outcomes; this could highlight variances in survival rates by region. This might also

138 support the healthcare system, public health control measures, and clinical practices to advance

139 the clinical outcomes in healthcare facilities by prioritizing high-risk regions and patient groups.

140 This study aimed to estimate the survival rates and hazard ratios (HRs) for MERS-CoV mortality

141 for 14- and 45-day post-symptom onset periods and assess spatial variations by both Saudi

142 Arabian regional and patient characteristics between 2012 and 2019.

\section{2. Materials and Methods}

144 2.1. MERS-CoV Data

145 All publicly available laboratory-confirmed cases of MERS-CoV reported between June 13, 146 2012, and April 30, 2019 were compiled by the Saudi Ministry of Health (SMH) via their 
147 Command and Control Center (CCC) website (center 2019) and WHO (WHO 2019). With the 148 emergence of MERS-CoV in Saudi Arabia, the CCC was launched in 2014 to take immediate 149 proactive action against future public health challenges by way of continuous coordination and 150 monitoring. For the current study, the MERS-CoV data was thoroughly reviewed, and a range of 151 checks was performed to ensure the data were consistent, complete, and fit for the study. A data 152 dictionary was developed for the variables for each case of MERS-CoV, including gender, age, 153 underlying comorbidity, exposure to camels, healthcare status, outcome, date of symptom onset, 154 date of death, city of residence, province, and region. The variables were categorized according 155 to gender (male/female), age $(<20,20-40,41-60$, or $\geq 61)$, comorbidity (yes/no), exposure to 156 camels (yes/no), HCW (yes/no), regions (five categories as shown below), provinces (13 157 categories as shown below), and outcome (alive/dead). The data contained no reported 158 comorbidity variable for 58 patients and no exposure to camel variable for 939 patients (713 159 were unknown or missing a source of infection and 226 were primary or secondary cases without 160 camel exposure status). The MERS-CoV data had been reported by Saudi city, so the data were 161 aggregated for the following 13 provinces, comprising five regions: Riyadh and Qassim (Central 162 region); Tabuk, Jouf, and Hail (North region); Assir, Baha, Jazan, and Najran (South region); the 163 Eastern province (East region); and Makkah and Madinah (West region). A spatial database of MERS-CoV incidence and mortality in Saudi Arabia was created using geographical information systems (GIS).

\subsection{Statistical Analysis}

167 A comparative epidemiological analysis using a Chi-square test was undertaken to determine the significant differences between the demographic and clinical characteristics of the survival and mortality rates of MERS-CoV cases at the regional level in Saudi Arabia. The variables used in the analysis included age and gender and whether the patients were HCWs, demonstrated underlying comorbidities, and had been exposed to camels. The statistical analyses were

172 performed using MedCalc software, Version 19.0.6, and a P-value $<0.05$ was considered 173 statistically significant. 
175 Survival analysis aims to model and analyze time-to-event data, that is, a dataset of the time that 176 an event occurred as its endpoint. In the current study, two MERS-CoV parameters, the time of 177 symptom onset and the date of death, were evaluated for 14 days and 45 days after the 178 appearance of symptoms. The incubation period of MERS-CoV, the elapsed time between 179 contracting the virus and displaying symptoms, ranged from 2 to 14 days (Ahmed 2018). The 14180 day period is medically important because it indicates the point at which most MERS-CoV 181 patients typically experience symptoms. The 45-day period was chosen to exhibit the progression 182 of MERS-CoV for an advanced period. The 14-day and 45-day MERS-CoV survival rates were 183 estimated using the Kaplan-Meier estimator at both the national and regional levels in Saudi 184 Arabia. The log-rank test was used to compare survival curves according to the demographic and 185 clinical factors affecting MERS-CoV patients. However, the log-rank test delivers a statistical 186 but not clinical appraisal of the effect of the factors (Bradburn et al. 2003). Thus, the Cox 187 proportional-hazards $(\mathrm{CPH})$ model was applied to assess the survival of MERS-CoV patients 188 regarding several concurrent factors and estimate the extent of the influence of each constituent factor. The CPH model is broadly applicable and is the most widely used multivariate approach for survival analysis (Bradburn et al. 2003). The CPH model examines the relationships of covariates to the time-to-event as expressed by the hazard function. The $\mathrm{CPH}$ model is interpreted using HRs, which are expressed as the ratio of the estimated hazard function under two different values of a covariate (George et al. 2014). An HR above one indicates that the event (in this case, death resulting from MERS-CoV) has a higher likelihood of occurring and that the covariate is positively related to the event probability and negatively related to the duration of survival. An HR less than one indicates that an event has a lower probability of occurring, and an HR of one indicates that the covariate does not influence the hazard of an event.

The probability of the endpoint (i.e. time to death) is termed the hazard, and the hazard is modeled as a group of predictor variables. The predictors (risk factor) are dichotomous and coded as 1 if present (died) and 0 if absent (alive). The value of the hazard ratio can then be inferred as the instant relative risk of an event at any time for a patient with the risk factor present compared to a patient with the risk factor absent, given both patients are equivalent for all other predictors. A variable is removed from the model if its related significance level is larger than the P-value (0.05). Survival time is the variable of the time to reach the event of 
206 interest (the outcome, in our case, death). The endpoint variable represents the occurrence of the

207 event (i.e. death) and comprises patient cases that have reached the endpoint (coded as 1) or

208 cases that have not reached the endpoint (coded as 0 ) as they are removed from the study (in our

209 case, at 14 and 45 days). The predictor variables are the variables we expect to predict survival

210 time (gender, age, underlying comorbidity, exposure to camels, healthcare status, region, and

211 province) (MedCalc 2020). When building our model, we applied a filter for particular regions

212 and provinces.

213 Using MedCalc software, the CPH model was applied to estimate the HRs and 95\%

214 confidence intervals (CIs) for the predictors of the two primary endpoints: 14- and 45-day

215 mortality. Both univariate and multivariate analyses were applied using the CPH model. The

216 former describes the MERS-CoV survival rate regarding the factor under examination but

217 disregards effects from any other factors; whereas, the latter simultaneously considers several

218 covariates that may affect patients' prognoses to enable better clinical assessment. The variables

219 used in the CPH model included a patient's age and gender and whether the patient was an

$220 \mathrm{HCW}$, had a pre-existing illness, and had been exposed to camels.

221 The MERS-CoV mortality risk factors for the 14- and 45-day post-symptom periods were

222 estimated for national, regional, and provincial levels in Saudi Arabia, and the spatial variations

223 were assessed according to region, province, and mortality hazard factors using both univariate

224 and multivariate $\mathrm{CPH}$ model analyses. For each post-symptom period, ten CPH models were

225 built: national, each region and province separately, incorporating regions as a covariate, and

226 incorporating provinces as a covariate for both the univariate and multivariate model analyses.

227 The regional and provincial factors characterized the geographical representation, following a 228 previously used approach (Ayele et al. 2017) for a survival analysis of mortality using the CPH 229 model.

230

231

232

233

234

235

\subsection{Spatial Analysis}

The spatial variations of the risk factors associated with MERS-CoV mortality were assessed using univariate local spatial autocorrelation and multivariate spatial clustering analyses. Spatial autocorrelation measures the association within values across locations (Getis 2008). It is the dependence of a particular variable's values on the values of the same variable recorded at nearby locations (Fortin MJ.; and Dale 2005). We applied the Getis-Ord Gi* statistic, a local 
236 spatial autocorrelation that detects the locations of statistically significant hot spots (the 237 clustering of high values) and cold spots (the clustering of low values) within the setting of 238 nearby features (Ord JK. and Getis 1995) using Esri ArcGIS 10.7.1. Given a set of provinces and 239 associated risk factor values, the Gi* statistic assesses whether the expressed pattern is high240 value cluster or low-value cluster (Mitchell 2005). To consider the spatial interaction between 241 the outcome and covariates examined in this study, we applied the Bivariate Local Moran Index 242 via GeoDa package. In principle, this index identifies the association between the value for one 243 variable at a location and the average of the neighboring values for another variable, i.e., its 244 spatial lag (Anselin et al. 2006).

245 Clustering is an unsupervised machine learning approach that involves determining natural 246 groupings within data (Duque JC 2007), and the K-means algorithm is one of the most popular 247 methods for clustering data (Spielman SE. and Thill 2008). We used multivariate spatial 248 clustering analysis to identify clusters of Saudi provinces according to patients' characteristics 249 variables for MERS-CoV mortality using the K-means algorithm via Esri ArcGIS. The resulting 250 maps created distinct clusters of variables (demographic, occupational, and clinical patient 251 characteristics for MERS-CoV mortality); whereby, the provinces that were part of a cluster 252 were as similar as possible and the provinces between clusters were as dissimilar as possible. 253 Each variable's contribution, evaluated using $\mathrm{r}^{2}$ values, was used to differentiate the provinces, 254 which revealed how much of the variation in the original data was kept after the clustering 255 process: the higher the $\mathrm{r}^{2}$ value for a specific variable, the better that variable was for discerning 256 the provinces (Pimpler 2017).

\section{3. Results}

\subsection{Characteristics of MERS-CoV Patients}

259

The analysis of the relationship of MERS-CoV mortality to patients' characteristics is shown in Table 1. A total of 2,037 laboratory-confirmed cases of human infection with MERS-CoV

261 were reported in Saudi Arabia between June 13, 2012, and April 30, 2019. Among these, 760 262 cases died (37.30\%), with an overall crude mortality rate of 2.37 per 100,000 population. A 263 higher number of deaths resulted from MERS-CoV cases involving males $(\mathrm{n}=558 ; 73.42 \%)$ 264 compared to females $(n=202 ; 26.58 \%)$; a statistically significant difference $(p=0.0007)$. The 
265 overall median age of the deceased cases was 62 years (interquartile range $=50-73$ ), ranging 266 from 2 to 114 years, while the mean age of the cases was 60.36, with a standard deviation of $267 \pm 17.99$.

268 The greatest number of deaths occurred among elderly patients (age $\geq 61$ ), representing $57.13 \%(\mathrm{n}=434)$ of the total deceased cases, which was statistically significant $(\mathrm{p}<0.0001)$.

270

271

272

273

274

275

276

277

278

279

280

281

282

283

284

285

286

287

288

289

290

291

292 MERS-CoV mortality was higher among non-HCWs $(\mathrm{n}=729 ; 95.93 \%)$ than among HCWs $(\mathrm{n}=$ $31 ; 4.07 \%)$, and this difference was statistically significant $(\mathrm{p}<0.0001)$. MERS-CoV patients with underlying comorbidities had a higher risk for mortality compared to those without $(\mathrm{n}=696$ [94.18\%] vs. $\mathrm{n}=43[5.82 \%] ; \mathrm{p}<0.0001)$. A lower mortality rate was found among those exposed to camels compared to those not exposed, although this difference was not statistically significant $(\mathrm{p}=0.3651)$. The Central region reported 366 deaths resulting from MERS-CoV, accounting for $48.16 \%$ of the total number of MERS-CoV deaths, followed by the West region ( $n=220$, with $28.95 \%$ mortality) and the East region $(n=98$, with $12.89 \%$ mortality), but the variation across regions was not statistically significant $(\mathrm{p}=0.7706)$.

\subsection{Spatial Pattern of MERS-CoV Survival}

Figure 1 displays the results of the spatial pattern of MERS-CoV survival in Saudi regions. Approximately $59.30 \%-65.25 \%$ of MERS-CoV cases across the Saudi regions were not fatal, but $34.75 \%-40.70 \%$ of the cases resulted in death, as shown in Figure 1A. The distribution of demographic, occupational, and clinical characteristics of MERS-CoV survival is shown in Figures 1B to 1F. MERS-CoV mortality was more frequent in males compared to females across all regions (ranging from $1.42 \%-12.47 \%$ for males and $0.20 \%-5.50 \%$ for females) out of the total number of MERS-CoV cases, as shown in Figure 1B. MERS-CoV mortality was significantly higher among elderly patients aged $\geq 61$ for all regions (ranging from $0.79 \%$ in the North region to $9.97 \%$ in the Central region), as shown in Figure 1C. MERS-CoV mortality was higher among non-HCWs $(1.57 \%-17.33 \%)$ than HCWs $(0.10 \%-0.64 \%)$ across all regions (see Figure 1D). A greater number of patients with underlying comorbidities $(1.52 \%-17.53 \%)$ died compared to those without $(0.10 \%-0.96 \%)$ across all regions (see Figure 1E). A higher mortality rate was found among MERS-CoV patients who had not been exposed to camels $(2.46 \%-$ 
$29323.32 \%)$ than those who had been exposed $(0.36 \%-6.56 \%)$ for all regions, as shown in Figure $2941 \mathrm{~F}$.

295

296

297

298

299

300

301

302

303

304

305

306

307

308

309

310

311

312

313

314

315

316

317

318

319

320

321

322

The spatial distribution of the total MERS-CoV mortality cases varied across the provinces, with most cases reported from Riyadh and Makkah followed by the Eastern and Qassim provinces (see Figure 2A). The spatial variation patterns of the risk factors, based on the demographic, occupational, and clinical characteristics of the MERS-CoV patients, also indicated spatially varied effects and were mainly elevated in Riyadh, Makkah, Eastern, and Qassim provinces but were degraded in the northern and southern provinces (see Figures 2B to $2 \mathrm{H}$ and Appendix Figures 1A to 1E). The univariate local spatial autocorrelation Getis-Ord Gi* analysis suggested that there were statistically significant hot spots in Riyadh province for all risk factors except for age $<20$, for which there were no hot spots across the entire country. Madinah province was a hot spot for the age 20-40 factor, and Riyadh and Madinah provinces were hot spots for the exposed to camel factor. Statistically significant cold spots were observed in Jouf province for all risk factors and detected for age $<20$, age 20-40, and patients without comorbidities in Jazan province. The bivariate spatial analysis with Bivariate Local Moran Index showed no statistically significant spatial autocorrelation between the examined covariates and MERS-CoV mortality attributable to $<20$ years and non-comorbidity in all provinces. However, the identification of such an autocorrelation for all the other covariates was observed in Riyadh province. The multivariate spatial clustering analysis applied here optimized within-cluster similarities and between-cluster differences for Saudi provinces based on the risk factors with subcategories (12 variables) for demographic, occupational, and clinical patient characteristics of MERS-CoV mortality. The results identified three spatial clusters of provinces, with the $\mathrm{r}^{2}$ for variables ranging between 0.85 and 0.97 . Clusters were found in Riyadh province (Cluster 1); Eastern, Makkah, and Qassim provinces (Cluster 2); and the remaining provinces in the north and south of the country (Cluster 3), see Appendix Figure 1F.

\subsection{Survival Analysis of MERS-CoV}

The mortality rate was $25.52 \%$ at 14 days, $32.35 \%$ at 45 days, and $37.30 \%$ overall. The survival rates for MERS-CoV at 14 and 45 days were estimated using the Kaplan-Meier method. These estimated survival rates were $70.20 \%$ (at 14 days), $57.70 \%$ (at 45 days), and $56.50 \%$ (overall). Figures 3 and 4 present the Kaplan-Meier curves for regional, demographic, 
323 occupational, and clinical factors for 14- and 45-day mortalities, respectively. The Central region $324(69.30 \%)$ had a lower 14-day survival rate than the East $(70.10 \%)$, North (70.20\%), West 325 (71.40\%), and South (72.70\%) regions, as shown in Figure 3A. For 45 days, the East region $326(56 \%)$ had a lower survival rate than the Central (57.30\%), West (58.70\%), South (60.30\%), and 327 North (70.20\%) regions (see Figure 4A). However, the differences in survival rates across all 328 Saudi regions were not statistically significant $(\mathrm{p}=0.9045$ and 0.9279 for 14 -day and 45-day 329 survival, respectively). Lower survival probabilities were found among males compared to 330 females (see Figures $3 \mathrm{~B}$ and $4 \mathrm{~B})(14$ days $=67.80 \%$ vs. $75 \%$, respectively, and 45 days $=$ $33154.10 \%$ vs. $65.20 \%$, respectively). Similarly, lower survival probabilities were found among the 332 elderly compared to younger people, see Figures 3C and 4C. Survival rates were also lower 333 among non-HCWs than HCWs (see Figures 3D and 4D): 14 days $=63.60 \%$ vs. 96.20\%, 334 respectively, and 45 days $=50.30 \%$ vs. 91.30\%, respectively. Survival rates were lower among 335 patients with comorbidities than among those without (see Figures 3E and 4E): 14 days = $33660.60 \%$ vs. 93\%, respectively, and 45 days $=47.50 \%$ vs. $89.60 \%$, respectively, and for patients 337 who were not exposed to camels compared to those who were exposed (see Figures $3 \mathrm{~F}$ and $4 \mathrm{~F}$ ): 33814 days $=69.40 \%$ vs. $73.10 \%$, respectively, and 45 days $=54 \%$ vs. $58.20 \%$, respectively.

339 Overall, the 14-day Kaplan-Meier curves for the factors of gender, age, HCW, presence of 340 comorbidities, and having been exposed to camels for each region showed relatively similar 341 patterns to those at the national level but with variations in the survival probabilities (see Figures $3425 \mathrm{~A}$ to $5 \mathrm{Y})$.

343

344

345

346

347

348

349

350

351

352

\subsection{Risk Assessment of MERS-CoV Mortality}

The MERS-CoV mortality risk factors for 14- and 45-day periods were estimated for the national level, each of the regions, and each of the provinces using both $\mathrm{CPH}$ modeled univariate and multivariate analyses. We also built models that incorporated regions and provinces as covariates.

The univariate risk factors for 14- and 45-day mortality are shown in Appendix Table 1. At the national level, during the 14-day mortality period, MERS-CoV survival was impaired according to the following HRs with varying statistical significance: males (1.34), age 41-60 (2.20), age $\geq 61$ (5.83), non-HCWs (17.61), and underlying comorbidities (7.21). During the 45day mortality period, similar risk factors were found compared to the 14-day period, but with 
353 relatively higher HRs for males and patients aged 41-60 and lower HRs for the other risk factors.

354 At the regional level, during the 14-day period and in all regions except the North and South 355 regions, patients aged $\geq 61(\mathrm{HR}=2.56-11.39)$ and those with underlying comorbidities $(\mathrm{HR}=$ 356 4.30-10.95) were associated with MERS-CoV mortality. In the Central $(\mathrm{HR}=9.47)$ and West 357 regions $(\mathrm{HR}=55.77)$, non-HCWs were linked to higher 14-day mortality rates, whereas being 358 male $(\mathrm{HR}=1.46)$ was only significant in the Central region. Overall, the univariate analysis for 359 45-day mortality identified similar risk factors as for the 14-day mortality, with relatively lower 360 HRs. However, non-HCWs $(H R=5.93)$ in the East region, males $(H R=1.40)$ in the West 361 region, and patients aged $\geq 61(\mathrm{HR}=2.43)$ in the North region were at higher risk for the 45-day 362 period but not for the 14-day period. Overall, the univariate analysis at the provincial level 363 identified similar risk factors as for the regional level (Appendix Table 2). When regions and 364 provinces were used as covariates in the CPH univariate models, only coming from Qassim 365 province was significantly associated with MERS-CoV mortality at 14 days $(\mathrm{HR}=2.12)$ 366 (Appendix Tables 3 and 4).

367 The results of the multivariate analysis for the national level and each of the regions are 368 shown in Table 2. The national model revealed that people aged $\geq 61(\mathrm{HR}=2.10)$, non-HCWs $369(\mathrm{HR}=10.12)$, and those with underlying comorbidities $(\mathrm{HR}=4.11)$ were independently related 370 to higher 14-day mortality $(\mathrm{p}<0.0001)$. The model for 45 -day mortality identified similar risk 371 factors to those of the 14-day model, but with an additional risk factor: patients aged 41-60 (HR $372=1.44)$. The regions showed variations in the mortality hazard models. Those aged $\geq 61(\mathrm{HR}=$ 373 1.86-5.39) and patients with underlying comorbidities ( $\mathrm{HR}=2.69-6.69)$ were independently 374 related to higher 14-day mortality in all regions except for the North and South regions. Non375 HCWs were significantly associated with mortality in the West $(\mathrm{HR}=29.34)$ and Central $(\mathrm{HR}=$ 376 4.55) regions, while those aged 41-60 were at risk only in the West region $(\mathrm{HR}=2.44)$. During 377 the 45-day mortality period, by region, similar risk factors were identified as for those in the 14378 day models, but with relatively lower HRs. The spatial distribution of the HRs for the MERS379 CoV risk factors for 14- and 45-day mortality based on multivariate analysis for each of the 380 regions and provinces are shown in Figure 6 and Appendix Figure 2, respectively. The estimated 381 HRs for 14- and 45-day mortality varied spatially by province. For the former, the highest HRs 382 were found in Makkah ( $\mathrm{HR}=2.1$ for age 41-60 and $\mathrm{HR}=27.03$ for non-HCWs), in Riyadh (HR $383=8.24$ for having comorbidity), and in Madinah $(\mathrm{HR}=8.59$ for age $\geq 61)$, while for the latter, the 
384 highest HRs were found in Makkah ( $\mathrm{HR}=3.52$ for age $\geq 61$ and $\mathrm{HR}=6.15$ for non-HCWs), in

385 Riyadh $(\mathrm{HR}=7.17$ for having comorbidity), and in Madinah $(\mathrm{HR}=11.14$ for age $41-60)(\mathrm{p}<$

386 0.0001). Overall, the multivariate analysis at the provincial level identified similar risk factors as

387 for the regional levels, as shown in Table 3, with the assistance to discover the specific risk

388 factor for each province. The results of the multivariate $\mathrm{CPH}$ modeling that incorporated regions

389 and provinces as covariates in addition to the other risk factors are shown in Tables 4 and 5.

390 Regionality was not significantly associated with 14- and 45-day mortality, while for the

391 provinces, coming from Makkah $(\mathrm{HRs}=1.30$ and 1.27) or Qassim (HRs $=1.77$ and 1.70)

392 provinces were independently related to higher 14- and 45-day mortality, respectively ( $\mathrm{p}<$ 393 0.0001).

394

395

396

397

398

399

400

401

402

403

404

405

406

407

408

409

410

411

412

413

\section{Discussion}

Saudi Arabia's MERS-CoV mortality rate is the highest in the world (Ahmed 2018; Park et al. 2018; WHO 2019) and has been examined by several Saudi Arabian studies (Assiri et al. 2013a; Mohd et al. 2016a). Nevertheless, little is known about the spatial variation of the survival rate and risk factors associated with MERS-CoV mortality across Saudi regions. This study first assessed whether there was a significant difference in MERS-CoV mortality related to demographic, occupational, and clinical characteristics. The survival rates and HRs for MERS$\mathrm{CoV}$ mortality were then estimated for 14- and 45-day post-symptom mortality periods, and the variations were assessed in relation to regions and risk factors.

The overall mortality rate in this study for all MERS-CoV patients in Saudi Arabia between 2012 and 2019 was estimated to be $37.30 \%$, similar to the previously reported $37 \%$ (Al Ghamdi et al. 2016) and comparable with previous studies estimating $36.5 \%$ or $38 \%$ (Coleman et al. 2017; Oboho et al. 2015). This rate is higher than the 10\%-34\% rates (Mohd et al. 2016b; Sherbini et al. 2017) and lower than the 40\%-65\% rates reported by other studies (Assiri et al. 2016; El Bushra et al. 2017; Feikin et al. 2015; Noorwali et al. 2015). These disparities might partially relate to sample size because most studies considered hospital-based cases reported by a specific hospital or referral hospital in a particular region. These differences can also be explained by the source of the MERS-CoV infection; some studies considered nosocomial outbreaks (Assiri et al. 2013b), while others considered household (Drosten et al. 2014) or overall cases (Alqahtani et al. 2018; Sherbini et al. 2017). At the Saudi regional level, variations 
414 in mortality rates ranged from $34.75 \%$ to $40.70 \%$, although the differences were not significant.

415 In South Korea, where the largest number of MERS-CoV outbreaks outside the Arabian

416 Peninsula occurred (Kim et al. 2019), one study that considered all MERS-CoV patients in South

417 Korea described a mortality rate of 20.4\% (Prevention 2015), lower than that of Saudi Arabia.

418 Although no vaccine or specific antiviral agents are currently available that can effectively

419 prevent or treat MERS-CoV, MERS-CoV mortality rates in Saudi Arabia have recently declined

420 from $33.81 \%$ in 2016 to $6.40 \%$ in 2019 . This might be attributable to the extensive control

421 measures implemented by the Saudi healthcare system in response to the MERS-CoV outbreak.

422 In this study, the estimated national survival rates for 14 days, 45 days, and overall were

$42370.20 \%, 57.70 \%$, and $56.50 \%$, respectively, while survival rates worldwide were $83.67 \%, 65.9 \%$,

424 and 69.04\%, respectively (Ahmed 2018), higher than those of Saudi Arabia. There are marginal

425 variations in survival rates across Saudi regions for the 14-day and 45-day periods $(70.10 \%-$

$42672.70 \%$ and $57.30 \%-70.20 \%$, respectively), but these were not statistically significant. Similar

427 survival rates across Saudi regions, particularly for the 14-day period, could be ascribed to the

428 simultaneous reporting of MERS-CoV symptoms and receiving a MERS-CoV diagnosis.

429 According to MERS-CoV patient characteristics, at the national level and among all regions,

430 mortality rates were higher among males, elderly patients aged $\geq 61$, patients with underlying

431 comorbidities, non-HCWs, and those not exposed to camels. Therefore, these groups increase the

432 odds of mortality resulting from MERS-CoV in Saudi Arabia and should be closely monitored.

433 The male-to-female ratio was estimated to be 2.76:1 for fatal MERS-CoV cases in Saudi

434 Arabia. The predominance of MERS-CoV cases among males in the current study is consistent

435 with previous studies from Saudi Arabia and South Korea (Chen et al. 2017; Sha et al. 2017).

436 Our findings suggest that there is also a statistically significant difference between male and

437 female mortality at the national level. This conforms with some previous research (Banik et al.

438 2016; Sherbini et al. 2017) but conflicts with other research (Ahmed 2017b; Alqahtani et al.

439 2018) that reported that males did not have a significantly higher risk of dying from MERS-CoV.

440 One study even suggested that survival rates were higher among males (Al Ghamdi et al. 2016),

441 although this study used a very small data sample. In the current study, MERS-CoV mortality

442 cases were found to be more frequent in males than females at the national level and for all

443 regions, and there was a statistically significant difference in the 14- and 45-day survival rates

444 between males and females at the national level and in the Central region but not in other 
445 regions. The univariate analysis showed that the hazard of 14- and 45-day mortality in MERS$446 \mathrm{CoV}$ male patients was higher than that for females only at the national level and in the Central 447 and West regions. This can be attributed to the high prevalence of MERS-CoV among males 448 who interact with camel-related activities in these regions (Gossner et al. 2016). In contrast, the 449 multivariate analysis could not find a significant gender effect at either the national or regional 450 level. Therefore, it is essential to examine the impact of gender on 14- and 45-day survival and 451 HRs across Saudi regions.

452 The analyses of the age distribution of MERS-CoV mortality showed that patients aged $\geq 61$ 453 most often died and had the lowest survival rates at the national and regional levels. According 454 to the univariate and multivariate analyses, being elderly was associated with a higher risk of 14455 and 45-day mortality in Saudi Arabia in general and in the Central, West, and East regions in 456 particular compared to those aged $<61$. These findings are consistent with previous studies 457 (Adegboye et al. 2017; Ahmed 2017b; Ahmed 2018; Alqahtani et al. 2018; Alsahafi \& Cheng 458 2016; Noorwali et al. 2015; Sherbini et al. 2017). The higher mortality rate of MERS-CoV in 459 aged patients might be explained by age-associated impairment of the immune system and the 460 presence of senescence-associated immune vulnerability among the elderly. This could amplify a person's susceptibility to and the progression of severe infections, increasing the likelihood of death (Aly et al. 2017). It may also be explained by the social and cultural customs that result in

463

464

465

466

467

468

469

470

471

472

473

474

475 suboptimal immune reactions following an exposure risk in which the elderly are involved, for example, camel-related activities and interactions (Omrani et al. 2013). A large proportion of aged patients also have comorbidities that may also impair immune functioning, thus affecting the likelihood of death from MERS-CoV (Webb et al. 2004).

In the current study and several others (Ahmed 2017b; Ahmed 2018; Alqahtani et al. 2018; Assiri et al. 2013b; Majumder et al. 2015; Nam et al. 2017; Sha et al. 2017; Sherbini et al. 2017), mortality from MERS-CoV was found to be significantly associated with underlying comorbidities. This is shown in the lower survival probability and higher HRs at 14 and 45 days among patients with comorbidities than in those without both at the national level and in most Saudi regions. The higher risk of death among patients with underlying diseases can partly be explained by higher viral loads in the respiratory tract and a longer duration of viral shedding compared to patients without such conditions (Sha et al. 2017). However, as the MERS-CoV data in this study were collected from publicly available sources, this study lacks information on 
476 the underlying causes of death and details of pre-existing conditions. Such information would be

477 valuable for recognizing specific pre-existing conditions related to higher mortality rates for 478 implementing measures that most effectively reduce fatalities.

479 It was also found that non-HCWs were linked to higher mortality rates, which is consistent 480 with other studies (Adegboye et al. 2017; Ahmed 2018; Rivers et al. 2016; Sha et al. 2017). 481 Lower survival rates and higher HRs for mortality for 14- and 45-day post-symptom periods 482 were also found among non-HCWs at the national level and in the Central, East, and West 483 regions. The lower survival rates and higher HRs for mortality among non-HCWs could be 484 explained by a lack of public awareness about the clinical signs and symptoms of the disease. 485 The early identification of MERS-CoV symptoms could result in early diagnosis and timely 486 treatment, leading to better rates of survival (Ahmed 2017a; Zumla et al. 2015). Higher MERS487 CoV survival rates among HCWs could be attributed to levels of knowledge, early diagnosis and 488 timely treatment, access to healthcare services, and adherence to control and prevention 489 guidelines.

490 Dromedary camels are likely a natural host for MERS-CoV. Transmission to humans can 491 occur through sporadic zoonotic infection after direct or indirect exposure to infected dromedary camels or their products (Azhar et al. 2014). However, this study's findings suggest that MERS-

$493 \mathrm{CoV}$ patients with a history of exposure to camels were not significantly associated with lower 494 survival at the national level or in the Saudi regions. One possible explanation for this 495 unexpected finding is non-reported data for the status of exposure to camels in the early stage of infection. However, similarly, another study (Sha et al. 2017) found no effect of exposure to camels on survival, either in the nosocomial or sporadic MERS-CoV cases in Saudi Arabia or South Korea.

The current study has revealed spatial variations in the risk factors for MERS-CoV mortality among regions and provinces. However, the bivariate spatial analysis discovered statistically significant spatial autocorrelation of high MERS-CoV mortality with high values for all covariates excluding $<20$ years and non-comorbidity in Riyadh province. Although the spatial pattern of each risk factor varies among the provinces, when they are combined based on the demographic, occupational, and clinical characteristics of MERS-CoV patients, three spatial clusters were identified: Riyadh (Cluster 1), Makkah, Eastern, and Qassim (Cluster 2), and other 
507 for particular provinces. The spatial pattern of the HRs for the significant risk factors was also 508 elevated in certain provinces, such as Riyadh, Makkah, Eastern, and Qassim. A possible 509 explanation for this could be differing provincial characteristics, such as healthcare systems, 510 quality of hospitalization practices, and accessibility to healthcare centers (Ahmed 2018; Feikin 511 et al. 2015). It can also be attributed to the spatial distribution at the provincial level for other 512 social, environmental, and health factors. The Riyadh (25\%, 23\%, and 25\%), Makkah (26\%, $51314 \%$, and $31 \%$ ), and Eastern $(15 \%, 18 \%$, and 13\%) provinces accounted for the highest

514 proportions in Saudi Arabia in terms of the total human population (Statistics 2010) and the 515 numbers of dromedary camels (Statistics 2015) and patients aged $\geq 15$ who suffer from chronic 516 disease (Statistics. 2017), respectively. Although the spatial levels of the analyses in this study 517 included national, regional $(n=5)$, and provincial $(n=13)$ levels, further research should be 518 conducted at lower spatial levels (e.g. cities $n=250$ and governorates $n=140$ ) and take into 519 account spatial correlation effects.

\section{Conclusions}

521 The MERS-CoV mortality rate in Saudi Arabia is the highest worldwide. However, no significant variations in MERS-CoV survival rates were found between Saudi regions and provinces. Being elderly, being a patient with underlying medical conditions, or being a non$\mathrm{HCW}$ were identified as risk factors for MERS-CoV mortality at the national level and in most Saudi regions and some provinces. The spatial pattern of the HRs for the significant risk factors was elevated in certain regions and provinces, and the reasons for this should be studied further. Detailed investigations at lower spatial levels should be undertaken to incorporate spatial correlation and dependency using an extended spatial hazard model for survival analysis, as reported by (Li et al. 2015) and (Zhou H. Hanson T. and Zhang 2020). The survival of MERS$\mathrm{CoV}$ patients could be improved by raising public awareness about the importance of earlysymptom notification and providing appropriate interventions, especially in aged MERS-CoV

532 patients and those with underlying conditions. Further investigation of specific pre-existing conditions linked to MERS-CoV mortality is also needed. 
537 Figure 1. Spatial distribution of MERS-CoV cases in Saudi regions between 2012 and 2019 by 538 (A) vital status, (B) gender, (C) age group, (D) healthcare worker, (E) comorbidity, and (F) 539 exposure to camels.

540

541 Figure 2. Spatial distribution of MERS-CoV mortality in Saudi provinces between 2012 and 5422019 by (A) overall, (B) male, (C) female, (D) age <20, (E) age 20-40, (F) age 41-60, (G) age $543 \geq 61$, and $(\mathrm{H})$ healthcare worker.

544

545 Figure 3. Survival rates for MERS-CoV for 14-day mortality at the Saudi national level between 5462012 and 2019 by (A) region, (B) gender, (C) age group, (D) healthcare worker, (E) comorbidity, 547 and (F) exposure to camels.

548

Figure 4. Survival rates for MERS-CoV for 45-day mortality at the Saudi national level between 550 2012 and 2019 by (A) region, (B) gender, (C) age group, (D) healthcare worker, (E) comorbidity, 551 and (F) exposure to camels.

552

Figure 5. Survival rates for MERS-CoV for 14-day mortality at the Saudi regional level between 554 2012 and 2019 by gender, age group, healthcare worker, comorbidity, and exposure to camels for the (A-E) Central, (F-J) East, (K-O) West, $(\mathrm{P}-\mathrm{T})$ North, and (U-Y) South regions.

556

Figure 6. Spatial distribution of hazard ratios for MERS-CoV mortality risk factors based on multivariate analysis for each region between 2012 and 2019 for 45-day mortality by (A) age 41-60, (B) age $\geq 61$, (C) non-healthcare worker, and (D) comorbidity. For 45-day mortality by (E) age 41-60, (F) age $\geq 61,(\mathrm{G})$ non-healthcare worker, and (H) comorbidity.

\section{Appendices}

563

564 Figure 1. Spatial distribution of MERS-CoV mortality in Saudi provinces between 2012 and 5652019 by (A) non-healthcare worker, (B) with comorbidity, (C) without comorbidity, (D) exposed 
566 to camels, (E) not exposed to camels, (F) multivariate spatial clusters for MERS-CoV mortality,

567 (G) MERS-CoV 14-day mortality, and (H) MERS-CoV 45-day mortality.

568

569 Figure 2. Spatial distribution of hazard ratios for MERS-CoV mortality risk factors based on 570 multivariate analysis for each region between 2012 and 2019 for 14-day mortality by (A) age 571 41-60, (B) age $\geq 61$, (C) non-healthcare worker, and (D) comorbidity. For 45-day mortality by 572 (E) age 41-60, (F) age $\geq 61,(\mathrm{G})$ non-healthcare worker, and (H) comorbidity.

573

574 Table 1. Risk factors and estimated hazard ratios for 14- and 45-day mortality for MERS-CoV 575 patients at the national level and each of the regions between 2012 and 2019 from univariate Cox 576 proportional-hazards $(\mathrm{CPH})$ modeling.

577

578 Table 2. Risk factors and estimated hazard ratios of 14- and 45-day mortality for MERS-CoV 579 patients for each of the provinces between 2012 and 2019 from univariate Cox proportional580 hazards $(\mathrm{CPH})$ modeling.

581

582 Table 3. Estimated hazard ratios for regions as covariates for 14- and 45-day mortality for 583 MERS-CoV patients between 2012 and 2019 from univariate Cox proportional-hazards (CPH) 584 modeling.

585

586 Table 4. Estimated hazard ratios for provinces as covariates for 14- and 45-day mortality for 587 MERS-CoV patients between 2012 and 2019 from univariate Cox proportional-hazards $(\mathrm{CPH})$ 588 modeling.

589

590 Acknowledgments: The authors wish to express their gratitude to King Abdulaziz City for 591 Science and Technology and King Faisal Specialist Hospital and Research Centre for supporting 592 this study, as well as the Saudi Ministry of Health and WHO for providing the data.

\section{References}


595

596

597

598

599

600

601

602

603

604

605

606

607

608

609

610

611

612

613

614

615

616

617

618

619

620

621

622

623

624

625

626

627

628

629

630

631

632
Adegboye OA, Gayawan E, and Hanna F. 2017. Spatial modelling of contribution of individual level risk factors for mortality from Middle East respiratory syndrome coronavirus in the Arabian Peninsula. PLoS One 12:e181215. 10.1371/journal.pone.0181215

Ahmed AE. 2017a. Diagnostic delays in 537 symptomatic cases of Middle East respiratory syndrome coronavirus infection in Saudi Arabia. Int J Infect Dis 62:47-51. 10.1016/j.ijid.2017.07.008

Ahmed AE. 2017b. The predictors of 3- and 30-day mortality in 660 MERS-CoV patients. BMC Infect Dis 17:615. 10.1186/s12879-017-2712-2

Ahmed AE. 2018. Estimating survival rates in MERS-CoV patients 14 and 45 days after experiencing symptoms and determining the differences in survival rates by demographic data, disease characteristics and regions: a worldwide study. Epidemiol Infect 146:489-495. 10.1017/S095026881700293X

Al-Ahmadi K, Alahmadi S, and Al-Zahrani A. 2019. Spatiotemporal Clustering of Middle East Respiratory Syndrome Coronavirus (MERS-CoV) Incidence in Saudi Arabia, 2012-2019. Int J Environ Res Public Health 16. 10.3390/ijerph16142520

Al Ghamdi M, Alghamdi KM, Ghandoora Y, Alzahrani A, Salah F, Alsulami A, Bawayan MF, Vaidya D, Perl TM, and Sood G. 2016. Treatment outcomes for patients with Middle Eastern Respiratory Syndrome Coronavirus (MERS CoV) infection at a coronavirus referral center in the Kingdom of Saudi Arabia. BMC Infect Dis 16:174. 10.1186/s12879-016-1492-4

Almekhlafi GA, Albarrak MM, Mandourah Y, Hassan S, Alwan A, Abudayah A, Altayyar S, Mustafa M, Aldaghestani T, Alghamedi A, Talag A, Malik MK, Omrani AS, and Sakr Y. 2016. Presentation and outcome of Middle East respiratory syndrome in Saudi intensive care unit patients. Crit Care 20:123. 10.1186/s13054-016-1303-8

Alqahtani FY, Aleanizy FS, Ali El Hadi Mohamed R, Alanazi MS, Mohamed N, Alrasheed MM, Abanmy N, and Alhawassi T. 2018. Prevalence of comorbidities in cases of Middle East respiratory syndrome coronavirus: a retrospective study. Epidemiol Infect:1-5. 10.1017/S0950268818002923

Alsahafi AJ, and Cheng AC. 2016. The epidemiology of Middle East respiratory syndrome coronavirus in the Kingdom of Saudi Arabia, 2012-2015. Int J Infect Dis 45:1-4. 10.1016/j.ijid.2016.02.004

Aly M, Elrobh M, Alzayer M, Aljuhani S, and Balkhy H. 2017. Occurrence of the Middle East Respiratory Syndrome Coronavirus (MERS-CoV) across the Gulf Corporation Council countries: Four years update. PLoS One 12:e183850. 10.1371/journal.pone.0183850

Anselin L, Syabri I, and Kho Y. 2006. GeoDa: An Introduction to Spatial Data Analysis. Geographical Analysis 38:5-22. 10.1111/j.0016-7363.2005.00671.x

Assiri A, Abedi GR, Bin Saeed AA, Abdalla MA, al-Masry M, Choudhry AJ, Lu X, Erdman DD, Tatti K, Binder AM, Rudd J, Tokars J, Miao C, Alarbash H, Nooh R, Pallansch M, Gerber SI, and Watson JT. 2016. Multifacility Outbreak of Middle East Respiratory Syndrome in Taif, Saudi Arabia. Emerg Infect Dis 22:32-40. 10.3201/eid2201.151370

Assiri A, Al-Tawfiq JA, Al-Rabeeah AA, Al-Rabiah FA, Al-Hajjar S, Al-Barrak A, Flemban H, Al-Nassir WN, Balkhy HH, AlHakeem RF, Makhdoom HQ, Zumla AI, and Memish ZA. 2013a. Epidemiological, demographic, and clinical characteristics of 47 cases of Middle East respiratory syndrome coronavirus disease from Saudi Arabia: a descriptive study. Lancet Infect Dis 13:752-761. 10.1016/S1473-3099(13)70204-4

Assiri A, McGeer A, Perl TM, Price CS, Al Rabeeah AA, Cummings DA, Alabdullatif ZN, Assad M, Almulhim A, Makhdoom H, Madani H, Alhakeem R, Al-Tawfiq JA, Cotten M, Watson SJ, Kellam P, Zumla AI, Memish ZA, and Team KM-CI.

Peer) reviewing PDF | (2020:01:44577:2:0:NEW 24 Jul 2020) 
633

634

635

636

637

638

639

640

641

642

643

644

645

646

647

648

649

650

651

652

653

654

655

656

657

658

659

660

661

662

663

664

665

666

667

668

669

670 2013b. Hospital outbreak of Middle East respiratory syndrome coronavirus. $N$ Engl J Med 369:407-416. 10.1056/NEJMoa1306742

Ayele DG, Zewotir TT, and Mwambi H. 2017. Survival analysis of under-five mortality using Cox and frailty models in Ethiopia. J Health Popul Nutr 36:25. 10.1186/s41043-017-0103-3

Azhar EI, El-Kafrawy SA, Farraj SA, Hassan AM, Al-Saeed MS, Hashem AM, and Madani TA. 2014. Evidence for camel-tohuman transmission of MERS coronavirus. N Engl J Med 370:2499-2505. 10.1056/NEJMoa1401505

Banik GR, Alqahtani AS, Booy R, and Rashid H. 2016. Risk factors for severity and mortality in patients with MERS-CoV: Analysis of publicly available data from Saudi Arabia. Virol Sin 31:81-84. 10.1007/s12250-015-3679-z

Bradburn MJ, Clark TG, Love SB, and Altman DG. 2003. Survival analysis part II: multivariate data analysis--an introduction to concepts and methods. Br J Cancer 89:431-436. 10.1038/sj.bjc.6601119

Breban R, Riou J, and Fontanet A. 2013. Interhuman transmissibility of Middle East respiratory syndrome coronavirus: estimation of pandemic risk. Lancet 382:694-699. 10.1016/S0140-6736(13)61492-0

center MoHiSACac. 2019. Available online: https://www.moh.gov.sa/en/CCC/Pages/default.aspx (accessed on 30 April 2019).

Chen X, Chughtai AA, Dyda A, and MacIntyre CR. 2017. Comparative epidemiology of Middle East respiratory syndrome coronavirus (MERS-CoV) in Saudi Arabia and South Korea. Emerg Microbes Infect 6:e51. 10.1038/emi.2017.40

Coleman CM, Sisk JM, Halasz G, Zhong J, Beck SE, Matthews KL, Venkataraman T, Rajagopalan S, Kyratsous CA, and Frieman MB. 2017. CD8+ T Cells and Macrophages Regulate Pathogenesis in a Mouse Model of Middle East Respiratory Syndrome. J Virol 91. 10.1128/JVI.01825-16

Cowling BJ, Park M, Fang VJ, Wu P, Leung GM, and Wu JT. 2015. Preliminary epidemiological assessment of MERS-CoV outbreak in South Korea, May to June 2015. Euro Surveill 20:7-13. 10.2807/1560-7917.es2015.20.25.21163

Das KM, Lee EY, Al Jawder SE, Enani MA, Singh R, Skakni L, Al-Nakshabandi N, AlDossari K, and Larsson SG. 2015. Acute Middle East Respiratory Syndrome Coronavirus: Temporal Lung Changes Observed on the Chest Radiographs of 55 Patients. AJR Am J Roentgenol 205:W267-274. 10.2214/AJR.15.14445

Drosten C, Meyer B, Muller MA, Corman VM, Al-Masri M, Hossain R, Madani H, Sieberg A, Bosch BJ, Lattwein E, Alhakeem RF, Assiri AM, Hajomar W, Albarrak AM, Al-Tawfiq JA, Zumla AI, and Memish ZA. 2014. Transmission of MERScoronavirus in household contacts. N Engl J Med 371:828-835. 10.1056/NEJMoa1405858

Duque JC RR, and Suriñach J. 2007. Supervised regionalization methods: A survey. Int Reg Sci Rev 30:195-220.

El Bushra HE, Al Arbash HA, Mohammed M, Abdalla O, Abdallah MN, Al-Mayahi ZK, Assiri AM, and BinSaeed AA. 2017. Outcome of strict implementation of infection prevention control measures during an outbreak of Middle East respiratory syndrome. Am J Infect Control 45:502-507. 10.1016/j.ajic.2016.12.020

Feikin DR, Alraddadi B, Qutub M, Shabouni O, Curns A, Oboho IK, Tomczyk SM, Wolff B, Watson JT, and Madani TA. 2015. Association of Higher MERS-CoV Virus Load with Severe Disease and Death, Saudi Arabia, 2014. Emerg Infect Dis 21:2029-2035. 10.3201/eid2111.150764

Fortin MJ.; and Dale M. 2005. Spatial analysis: a guide for ecologists. Cambridge, Cambridge University Press:365.

George B, Seals S, and Aban I. 2014. Survival analysis and regression models. J Nucl Cardiol 21:686-694. 10.1007/s12350-0149908-2

Getis A. 2008. A History of the Concept of Spatial Autocorrelation: A Geographer's Perspective. Geographical Analysis 40:297309. 10.1111/j.1538-4632.2008.00727.x

Peer] reviewing PDF | (2020:01:44577:2:0:NEW 24 Jul 2020) 
671 Gossner C, Danielson N, Gervelmeyer A, Berthe F, Faye B, Kaasik Aaslav K, Adlhoch C, Zeller H, Penttinen P, and Coulombier

672 D. 2016. Human-Dromedary Camel Interactions and the Risk of Acquiring Zoonotic Middle East Respiratory

673

674

675

676

677

678

679

680

681

682

683

684

685

686

687

688

689

690

691

692

693

694

695

696

697

698

699

700

701

702

703

704

705

706

707

Syndrome Coronavirus Infection. Zoonoses Public Health 63:1-9. 10.1111/zph.12171

Hui DS, Azhar EI, Kim YJ, Memish ZA, Oh MD, and Zumla A. 2018. Middle East respiratory syndrome coronavirus: risk factors and determinants of primary, household, and nosocomial transmission. Lancet Infect Dis 18:e217-e227. $10.1016 / \mathrm{S} 1473-3099(18) 30127-0$

Kim YS, Aigerim A, Park U, Kim Y, Rhee JY, Choi JP, Park WB, Park SW, Kim Y, Lim DG, Inn KS, Hwang ES, Choi MS, Shin HS, and Cho NH. 2019. Sequential Emergence and Wide Spread of Neutralization Escape Middle East Respiratory Syndrome Coronavirus Mutants, South Korea, 2015. Emerg Infect Dis 25:1161-1168. $10.3201 /$ eid2506.181722

Lessler J, Salje H, Van Kerkhove MD, Ferguson NM, Cauchemez S, Rodriquez-Barraquer I, Hakeem R, Jombart T, Aguas R, AlBarrak A, Cummings DA, Scenario ME-C, and Modeling Working G. 2016. Estimating the Severity and Subclinical Burden of Middle East Respiratory Syndrome Coronavirus Infection in the Kingdom of Saudi Arabia. Am J Epidemiol 183:657-663. 10.1093/aje/kwv452

Li L, Hanson T, and Zhang J. 2015. Spatial extended hazard model with application to prostate cancer survival. Biometrics 71:313-322. 10.1111/biom. 12268

Liu M, Jiang C, Donovan C, Wen Y, and Sun W. 2015. Middle East Respiratory Syndrome and Medical Students: Letter from China. Int J Environ Res Public Health 12:13289-13294. 10.3390/ijerph121013289

Majumder MS, Kluberg SA, Mekaru SR, and Brownstein JS. 2015. Mortality Risk Factors for Middle East Respiratory Syndrome Outbreak, South Korea, 2015. Emerg Infect Dis 21:2088-2090. 10.3201/eid2111.151231

MedCalc. 2020. MedCalc Statistical Software version 19.2.6. MedCalc Software Ltd, Ostend, Belgium; https://wwwmedcalcorg.

Mitchell A. 2005. The ESRI guide to GIS analysis, spatial measurements and statistics. ESRI, Redlands, CA.

Mizumoto K, Saitoh M, Chowell G, Miyamatsu Y, and Nishiura H. 2015. Estimating the risk of Middle East respiratory syndrome (MERS) death during the course of the outbreak in the Republic of Korea, 2015. Int J Infect Dis 39:7-9. 10.1016/j.ijid.2015.08.005

Mohd HA, Al-Tawfiq JA, and Memish ZA. 2016a. Middle East Respiratory Syndrome Coronavirus (MERS-CoV) origin and animal reservoir. Virol J 13:87. 10.1186/s12985-016-0544-0

Mohd HA, Memish ZA, Alfaraj SH, McClish D, Altuwaijri T, Alanazi MS, Aloqiel SA, Alenzi AM, Bafaqeeh F, Mohamed AM, Aldosari K, and Ghazal S. 2016b. Predictors of MERS-CoV infection: A large case control study of patients presenting with ILI at a MERS-CoV referral hospital in Saudi Arabia. Travel Med Infect Dis 14:464-470. 10.1016/j.tmaid.2016.09.008

Nam HS, Park JW, Ki M, Yeon MY, Kim J, and Kim SW. 2017. High fatality rates and associated factors in two hospital outbreaks of MERS in Daejeon, the Republic of Korea. Int J Infect Dis 58:37-42. 10.1016/j.ijid.2017.02.008

Noorwali AA, Turkistani AM, Asiri SI, Trabulsi FA, Alwafi OM, Alzahrani SH, Rashid MM, Hegazy SA, Alzaydi MD, and Bawakid KO. 2015. Descriptive epidemiology and characteristics of confirmed cases of Middle East respiratory syndrome coronavirus infection in the Makkah Region of Saudi Arabia, March to June 2014. Ann Saudi Med 35:203209. $10.5144 / 0256-4947.2015 .203$

Peer) reviewing PDF | (2020:01:44577:2:0:NEW 24 Jul 2020) 
708

709

710

711

712

713

714

715

716

717

718

719

720

721

722

723

724

725

726

727

728

729

730

731

732

733

734

735

736

737

738

739

740

741

742

743

744

745

746

Oboho IK, Tomczyk SM, Al-Asmari AM, Banjar AA, Al-Mugti H, Aloraini MS, Alkhaldi KZ, Almohammadi EL, Alraddadi BM, Gerber SI, Swerdlow DL, Watson JT, and Madani TA. 2015. 2014 MERS-CoV outbreak in Jeddah--a link to health care facilities. $N$ Engl J Med 372:846-854. 10.1056/NEJMoa1408636

Omrani AS, Matin MA, Haddad Q, Al-Nakhli D, Memish ZA, and Albarrak AM. 2013. A family cluster of Middle East Respiratory Syndrome Coronavirus infections related to a likely unrecognized asymptomatic or mild case. Int $J$ Infect Dis 17:e668-672. 10.1016/j.ijid.2013.07.001

Ord JK. and Getis A. 1995. Local spatial autocorrelation statistics: distributional issues and an application. Geogr Anal p286306.

Park JE, Jung S, Kim A, and Park JE. 2018. MERS transmission and risk factors: a systematic review. BMC Public Health 18:574. 10.1186/s12889-018-5484-8

Penttinen PM, Kaasik-Aaslav K, Friaux A, Donachie A, Sudre B, Amato-Gauci AJ, Memish ZA, and Coulombier D. 2013. Taking stock of the first 133 MERS coronavirus cases globally--Is the epidemic changing? Euro Surveill 18. 10.2807/1560-7917.es2013.18.39.20596

Pimpler E. 2017. Spatial analytics with ArcGIS. Birmingham, UK: Packt Publishing Ltd.

Prevention KCfDCa. 2015. Middle East respiratory syndrome coronavirus outbreak in the Republic of Korea. Osong Public Health Res Perspect 6:269-278.

Rivers CM, Majumder MS, and Lofgren ET. 2016. Risks of Death and Severe Disease in Patients With Middle East Respiratory Syndrome Coronavirus, 2012-2015. Am J Epidemiol 184:460-464. 10.1093/aje/kww013

Saad M, Omrani AS, Baig K, Bahloul A, Elzein F, Matin MA, Selim MA, Al Mutairi M, Al Nakhli D, Al Aidaroos AY, Al Sherbeeni N, Al-Khashan HI, Memish ZA, and Albarrak AM. 2014. Clinical aspects and outcomes of 70 patients with Middle East respiratory syndrome coronavirus infection: a single-center experience in Saudi Arabia. Int J Infect Dis 29:301-306. 10.1016/j.ijid.2014.09.003

Sha J, Li Y, Chen X, Hu Y, Ren Y, Geng X, Zhang Z, and Liu S. 2017. Fatality risks for nosocomial outbreaks of Middle East respiratory syndrome coronavirus in the Middle East and South Korea. Arch Virol 162:33-44. 10.1007/s00705-0163062-x

Sherbini N, Iskandrani A, Kharaba A, Khalid G, Abduljawad M, and Al-Jahdali H. 2017. Middle East respiratory syndrome coronavirus in Al-Madinah City, Saudi Arabia: Demographic, clinical and survival data. J Epidemiol Glob Health 7:2936. 10.1016/j.jegh.2016.05.002

Spielman SE. and Thill J. 2008. Social area analysis, data mining, and GIS. Comput Environ Urban Syst 32:110-122.

Statistics GAf. 2010. The general population and housing census in Saudi Arabia. Avilable at (https://wwwstatsgovsa/en/13).

Statistics GAf. 2015. Detailed results agricultural census in Saudi Arabia. Avilable at (https://wwwstatsgovsa/en/22).

Statistics. GAf. 2017. Family health survey in in Saudi Arabia. Avilable at (https://wwwstatsgovsa/en/965).

Webb GF, Blaser MJ, Zhu H, Ardal S, and Wu J. 2004. Critical role of nosocomial transmission in the toronto sars outbreak. Math Biosci Eng 1:1-13. 10.3934/mbe.2004.1.1

WHO. 2019. Emergencies: Middle East Respiratory Syndrome Coronavirus (MERS-CoV). Available online: https://www.who.int/emergencies/mers-cov/en/ (accessed on 27 April 2019).

Zaki AM, van Boheemen S, Bestebroer TM, Osterhaus AD, and Fouchier RA. 2012. Isolation of a novel coronavirus from a man with pneumonia in Saudi Arabia. N Engl J Med 367:1814-1820. 10.1056/NEJMoa1211721

Zhou H. Hanson T. and Zhang J. 2020. spBayesSurv: Fitting Bayesian Spatial Survival Models Using R. J Stat Softw 92:1-33.

Peer] reviewing PDF | (2020:01:44577:2:0:NEW 24 Jul 2020) 
747 Zumla A, Hui DS, and Perlman S. 2015. Middle East respiratory syndrome. Lancet 386:995-1007. 10.1016/S0140748 6736(15)60454-8

749 


\section{Table $\mathbf{1}$ (on next page)}

Table 1

Vital status of MERS-CoV patient characteristics in Saudi Arabia between 2012 and 2019. 
2 Table 1. Vital status of MERS-CoV patient characteristics in Saudi Arabia between 2012 and 2019.

3

\begin{tabular}{|c|c|c|c|c|}
\hline \multirow[t]{2}{*}{ Factors } & \multicolumn{2}{|c|}{ Vital status } & \multirow{2}{*}{$\begin{array}{l}\text { Total } \\
(\mathrm{n}=2,037)(\%)\end{array}$} & \multirow[t]{2}{*}{$\mathbf{P}$} \\
\hline & Alive $(\mathrm{n}=1,277,62.70 \%)$ & Dead $(n=760,37.30 \%)$ & & \\
\hline Gender & & & & 0.0007 \\
\hline Male & 846 (66.24\%) & $558(73.42 \%)$ & 1404 (68.92\%) & \\
\hline Female & $431(33.76 \%)$ & $202(26.58 \%)$ & $633(31.08 \%)$ & \\
\hline Age (years) & & & & $<0.0001$ \\
\hline$<20$ & $39(3.05 \%)$ & $10(1.31 \%)$ & $49(2.40 \%)$ & \\
\hline $20-40$ & $439(34.37 \%)$ & $82(10.78 \%)$ & $521(25.57 \%)$ & \\
\hline $41-60$ & $529(41.42 \%)$ & $234(30.78 \%)$ & $763(37.45 \%)$ & \\
\hline$\geq 61$ & $270(21.16 \%)$ & $434(57.13 \%)$ & $704(34.58 \%)$ & \\
\hline Healthcare worker & & & & $<0.0001$ \\
\hline Yes & $345(27.01 \%)$ & $31(4.07 \%)$ & $376(18.45 \%)$ & \\
\hline No & $932(72.99 \%)$ & 729 (95.93\%) & $1661(81.55 \%)$ & \\
\hline Comorbidity & & & & $<0.0001$ \\
\hline Yes & $780(62.90 \%)$ & $696(94.18 \%)$ & $1476(74.58 \%)$ & \\
\hline No & $460(37.10 \%)$ & $43(5.82 \%)$ & $503(25.42 \%)$ & \\
\hline Exposure to camels & & & & 0.3651 \\
\hline Yes & $157(22.81 \%)$ & $84(20.48 \%)$ & $241(21.94 \%)$ & \\
\hline No & $531(77.19 \%)$ & $326(79.52 \%)$ & $857(78.05 \%)$ & \\
\hline Region & & & & 0.7706 \\
\hline Central & $641(50.20 \%)$ & $366(48.16 \%)$ & 1007 (49.44\%) & \\
\hline East & $150(11.75 \%)$ & $98(12.98 \%)$ & $248(12.17 \%)$ & \\
\hline West & $358(28.03 \%)$ & $220(28.95 \%)$ & $578(28.38 \%)$ & \\
\hline North & $51(3.99 \%)$ & $35(4.16 \%)$ & $86(4.22 \%)$ & \\
\hline South & $77(6.03 \%)$ & $41(5.39 \%)$ & $118(5.79 \%)$ & \\
\hline
\end{tabular}

4

5

6

7

8

9 
11

12

Peer] reviewing PDF | (2020:01:44577:2:0:NEW 24 Jul 2020) 


\section{Table 2 (on next page)}

Table 2

Risk factors and estimated hazard ratios for 14- and 45-day mortality for MERS-CoV patients at the national level and each of the regions between 2012 and 2019 from multivariate Cox proportional-hazards (CPH) modeling 
2 Table 2. Risk factors and estimated hazard ratios for 14- and 45-day mortality for MERS-CoV 3 patients at the national level and each of the regions between 2012 and 2019 from multivariate Cox 4 proportional-hazards $(\mathrm{CPH})$ modeling.

5

\begin{tabular}{|c|c|c|c|c|c|c|}
\hline \multirow[b]{2}{*}{ Regions/Factors } & \multicolumn{3}{|c|}{ 14-day mortality } & \multicolumn{3}{|c|}{ 45-day mortality } \\
\hline & HR & $95 \%$ CI for HR & $\mathbf{P}$ & HR & 95\% CI for HR & $\mathbf{P}$ \\
\hline National & & & $<0.0001$ & & & $<0.0001$ \\
\hline Age $41-60$ years & NV & NV & NV & 1.44 & $1.12-1.86$ & 0.0040 \\
\hline Age $\geq 61$ years & 2.10 & $1.72-2.56$ & $<0.0001$ & 2.47 & $1.93-3.17$ & $<0.0001$ \\
\hline Non-healthcare worker & 10.12 & $4.75-21.52$ & $<0.0001$ & 4.45 & $2.90-6.81$ & $<0.0001$ \\
\hline With comorbidity & 4.11 & $2.75-6.14$ & $<0.0001$ & 3.55 & $2.56-4.93$ & $<0.0001$ \\
\hline Exposed to camel & 0.73 & $0.56-0.93$ & 0.0134 & 0.82 & $0.67-0.99$ & 0.0450 \\
\hline Central & & & $<0.0001$ & & & $<0.0001$ \\
\hline Age $\geq 61$ years & 1.86 & $1.41-2.46$ & $<0.0001$ & 1.74 & $1.38-2.19$ & $<0.0001$ \\
\hline Non-healthcare worker & 4.55 & $1.99-10.38$ & 0.0003 & 3.14 & $1.73-5.68$ & 0.0002 \\
\hline With comorbidity & 6.69 & $3.49-12.80$ & $<0.0001$ & 6.08 & $3.57-10.36$ & $<0.0001$ \\
\hline East & & & $<0.0001$ & & & $<0.0001$ \\
\hline Age $\geq 61$ years & 2.67 & 1.46 to 4.87 & 0.0013 & 1.95 & $1.24-3.05$ & 0.0034 \\
\hline With comorbidity & 2.69 & 1.00 to 7.23 & 0.0485 & 3.01 & $1.40-6.44$ & 0.0044 \\
\hline West & & & $<0.0001$ & & & $<0.0001$ \\
\hline Age $41-60$ years & 2.44 & $1.28-4.62$ & 0.0062 & 2.34 & $1.45-3.79$ & 0.0005 \\
\hline Age $\geq 61$ years & 5.39 & $2.91-9.96$ & $<0.0001$ & 4.80 & $2.97-7.74$ & $<0.0001$ \\
\hline Non-healthcare worker & 29.34 & $4.05-212.40$ & 0.0008 & 5.40 & $2.72-10.74$ & $<0.0001$ \\
\hline With comorbidity & 3.76 & $1.62-8.71$ & 0.0019 & 3.29 & $1.77-6.13$ & 0.0002 \\
\hline Exposed to camel & 0.52 & $0.29-0.92$ & 0.0254 & 0.67 & $0.45-0.98$ & 0.0436 \\
\hline North & & & & & & 0.0009 \\
\hline Without comorbidity & NV & NV & NV & 0.15 & $0.03-0.65$ & 0.0109 \\
\hline
\end{tabular}

$6 \quad \mathrm{NV}$ : No variables were retained in this model. Note: No variables were retained in this model for the South region.

7

8

9

10

11

12

13

14

15 


\section{Table 3 (on next page)}

Table 3

Table 3. Risk factors and estimated hazard ratios of 14- and 45-day mortality for MERS-CoV patients for each of the provinces between 2012 and 2019 from multivariate Cox proportional-hazards $(\mathrm{CPH})$ modeling 
2 Table 3. Risk factors and estimated hazard ratios of 14- and 45-day mortality for MERS-CoV 3 patients for each of the provinces between 2012 and 2019 from multivariate Cox proportional4 hazards $(\mathrm{CPH})$ modeling.

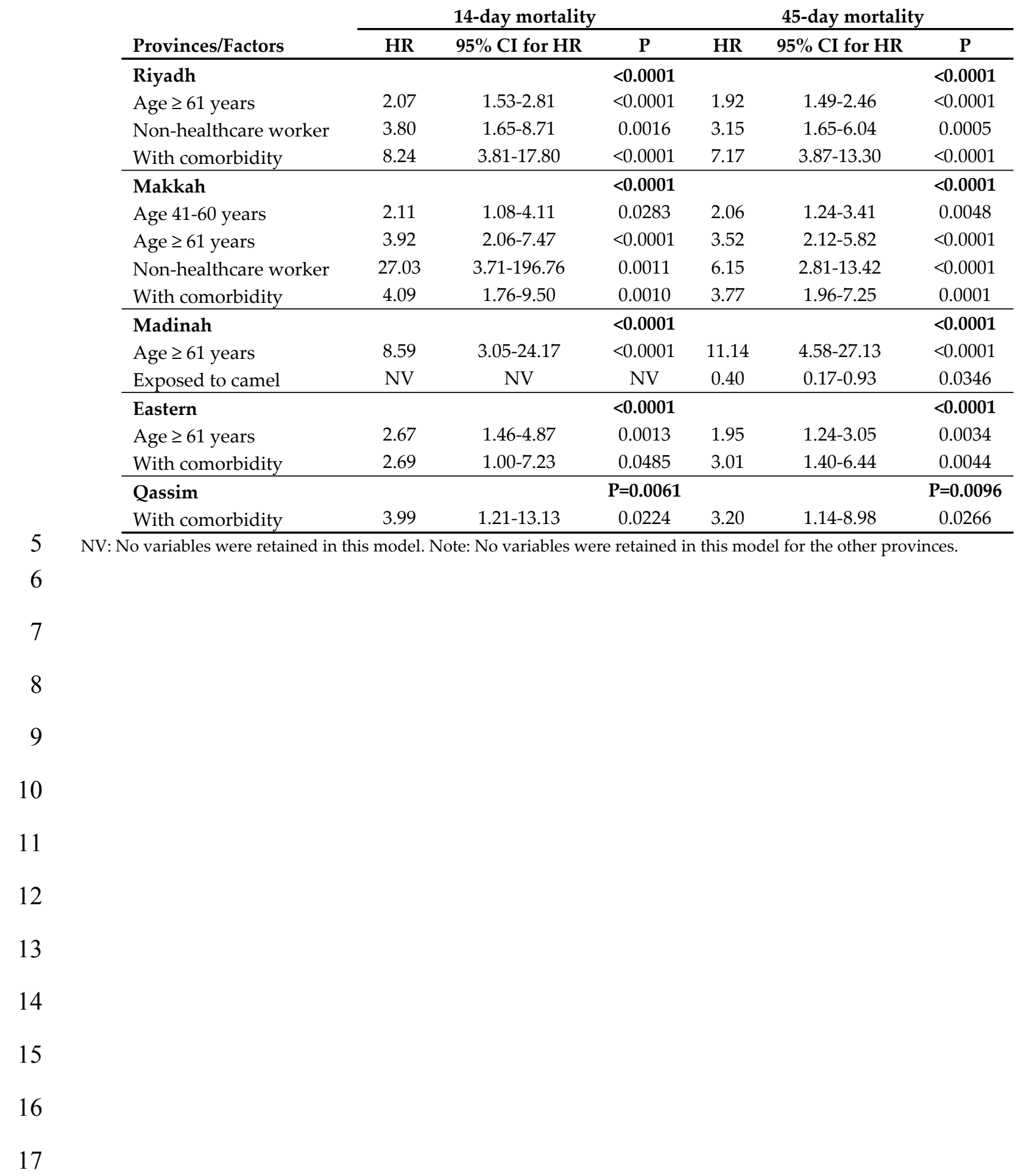




\section{Table 4 (on next page)}

\section{Table 4}

Survival rates for MERS-CoV for 45-day mortality at the Saudi national level between 2012 and 2019 by (A) region, (B) gender, (C) age group, (D) healthcare worker, (E) comorbidity, and $(F)$ exposure to camels. 
2 Table 4. Estimated hazard ratios for regions as covariates for 14- and 45-day mortality for MERS-CoV patients 3 between 2012 and 2019 from multivariate Cox proportional-hazards (CPH) modelling.

\begin{tabular}{lcccccc} 
& \multicolumn{3}{c}{ 14-day mortality } & \multicolumn{3}{c}{ 45-day mortality } \\
\cline { 2 - 7 } Factors & HR & $\mathbf{9 5 \%}$ CI for HR & P & HR & 95\% CI for HR & P \\
\hline & & & $<\mathbf{0 . 0 0 0 1}$ & & & $\mathbf{0 . 0 0 0 1}$ \\
Age 41-60 years & NV & NV & NV & 1.44 & $1.12-1.86$ & 0.0040 \\
Age $\geq$ 61 years & 2.10 & $1.72-2.56$ & $<0.0001$ & 2.47 & $1.93-3.17$ & $<0.0001$ \\
Non-healthcare worker & 10.12 & $4.75-21.52$ & $<0.0001$ & 4.45 & $2.90-6.81$ & $<0.0001$ \\
With comorbidity & 4.11 & $2.75-6.14$ & $<0.0001$ & 3.55 & $2.56-4.93$ & $<0.0001$ \\
Exposed to camel & 0.73 & $0.56-0.93$ & 0.0134 & 0.82 & $0.67-0.99$ & 0.0450 \\
\hline
\end{tabular}

4 NV: No variables were retained in this model. Note: No variables were retained in this model for the region factor.

5

6

7

8

9

10

11

12

13

14

15

16

17

18

19

20

21

22

23 


\section{Table 5 (on next page)}

Table 5

Estimated hazard ratios for provinces as covariates for 14- and 45-day mortality for MERSCoV patients between 2012 and 2019 from multivariate Cox proportional-hazards (CPH) modeling. 
2 Table 5. Estimated hazard ratios for provinces as covariates for 14- and 45-day mortality for MERS-CoV 3 patients between 2012 and 2019 from multivariate Cox proportional-hazards (CPH) modeling.

\begin{tabular}{lcccccc} 
& \multicolumn{3}{c}{ 14-day mortality } & \multicolumn{3}{c}{ 45-day mortality } \\
\cline { 2 - 7 } Factors & HR & $\mathbf{9 5 \%}$ CI for HR & $\mathbf{P}$ & HR & $\mathbf{9 5 \% ~ C I ~ f o r ~ H R ~}$ & $\mathbf{P}$ \\
\hline & & & $<\mathbf{0 . 0 0 0 1}$ & & & $<\mathbf{0 0 0 1}$ \\
Age 41-60 years & 1.39 & $1.01-1.91$ & 0.0400 & 1.47 & $1.14-1.89$ & 0.0028 \\
Age $\geq$ 61 years & 2.61 & $1.93-3.55$ & $<0.0001$ & 2.49 & $1.95-3.20$ & $<0.0001$ \\
Non-healthcare worker & 9.98 & $4.68-21.28$ & $<0.0001$ & 4.60 & $3.00-7.06$ & $<0.0001$ \\
With comorbidity & 3.78 & $2.51-5.68$ & $<0.0001$ & 3.52 & $2.54-4.88$ & $<0.0001$ \\
Exposed to camel & 0.72 & $0.56-0.93$ & 0.0121 & 0.82 & $0.67-0.99$ & 0.0043 \\
Makkah province & 1.30 & $1.22-2.55$ & 0.0226 & 1.27 & $1.06-1.52$ & 0.0081 \\
Qassim province & 1.77 & $0.56-0.93$ & 0.0023 & 1.70 & $1.24-2.33$ & 0.0009 \\
\hline
\end{tabular}

4

5

6

7

8

9 


\section{Figure 1}

Figure 1.

Spatial distribution of MERS-CoV cases in Saudi regions between 2012 and 2019 by (A) vital

status, (B) gender, (C) age group, (D) healthcare worker, (E) comorbidity, and (F) exposure to camels. 

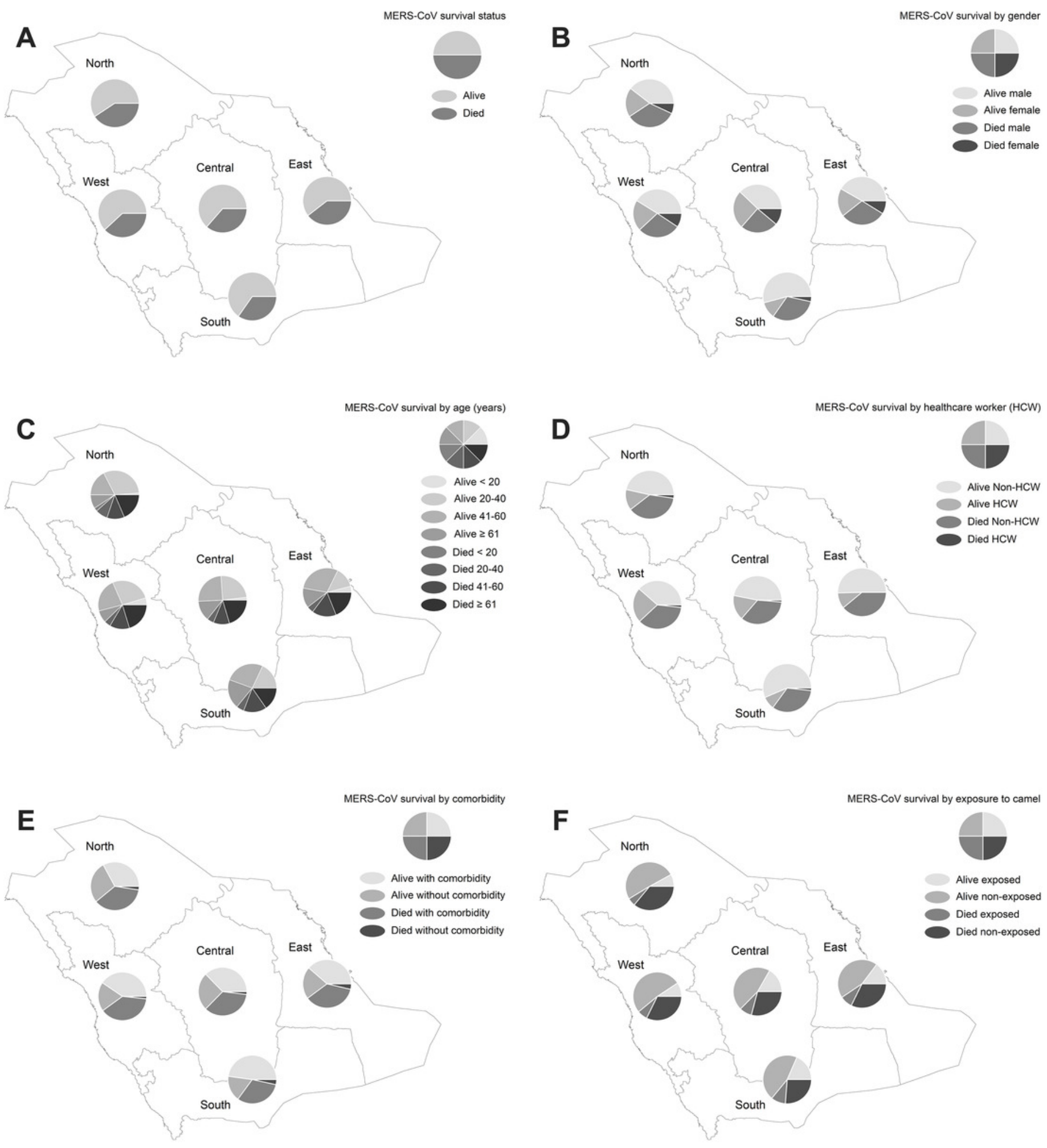
Figure 2

Figure 2

Spatial distribution of MERS-CoV mortality in Saudi provinces between 2012 and 2019 by (A)

overall, (B) male, (C) female, (D) age $<20$, (E) age 20-40, (F) age 41-60, (G) age $\geq 61$, and $(\mathrm{H})$ healthcare worker. 

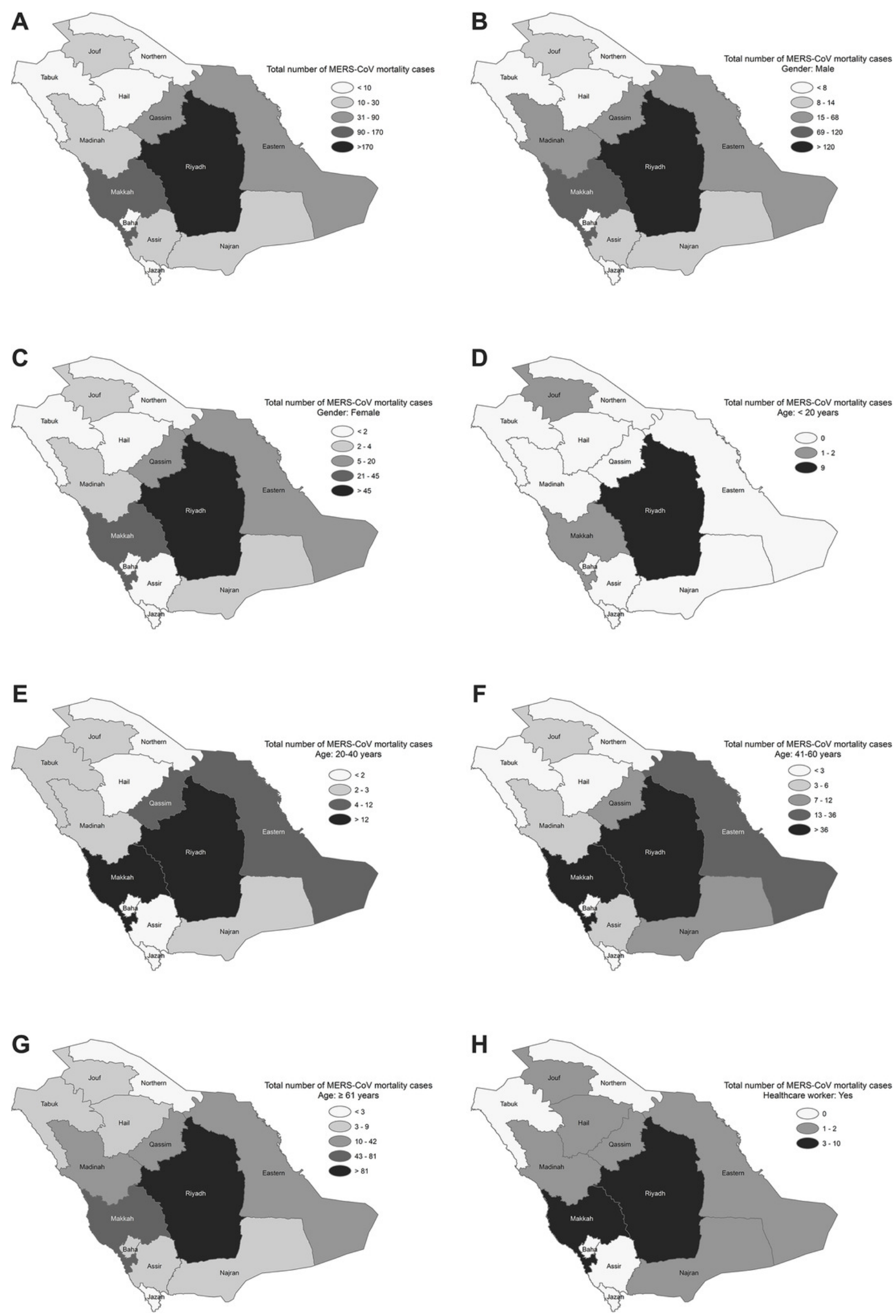
Figure 3

Figure 3

Survival rates for MERS-CoV for 14-day mortality at the Saudi national level between 2012 and 2019 by (A) region, (B) gender, (C) age group, (D) healthcare worker, (E) comorbidity, and $(F)$ exposure to camels. 
A

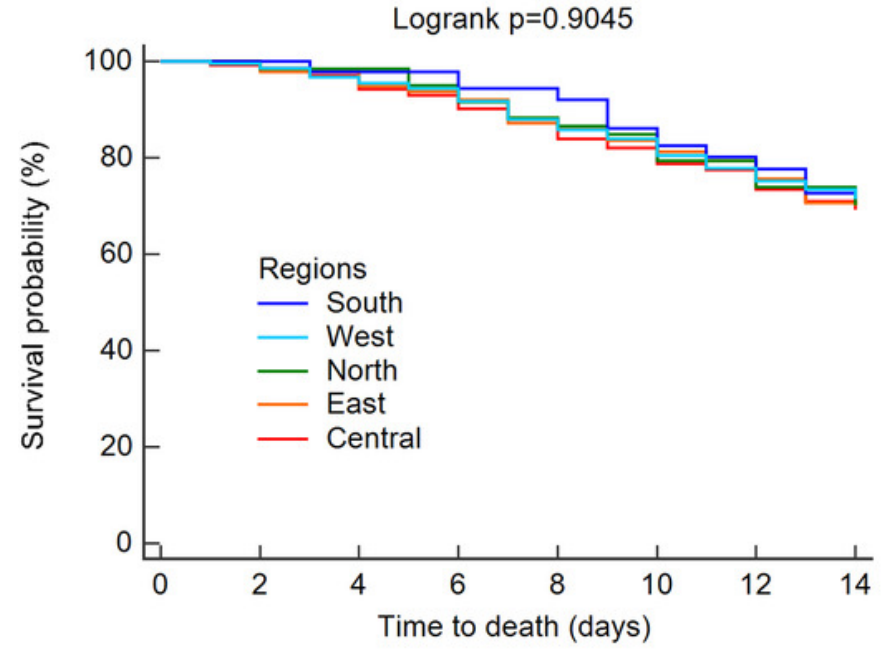

C

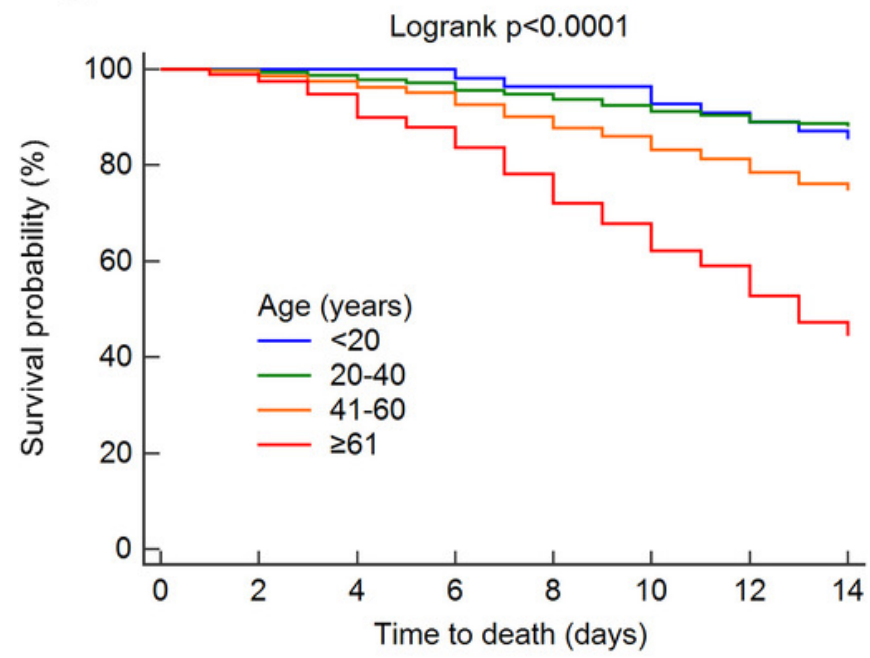

E

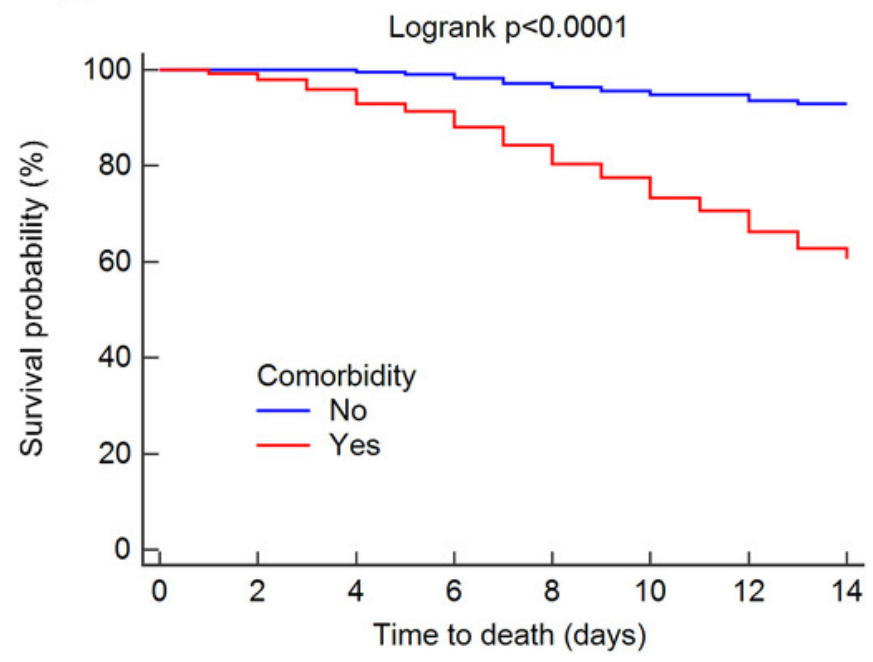

B

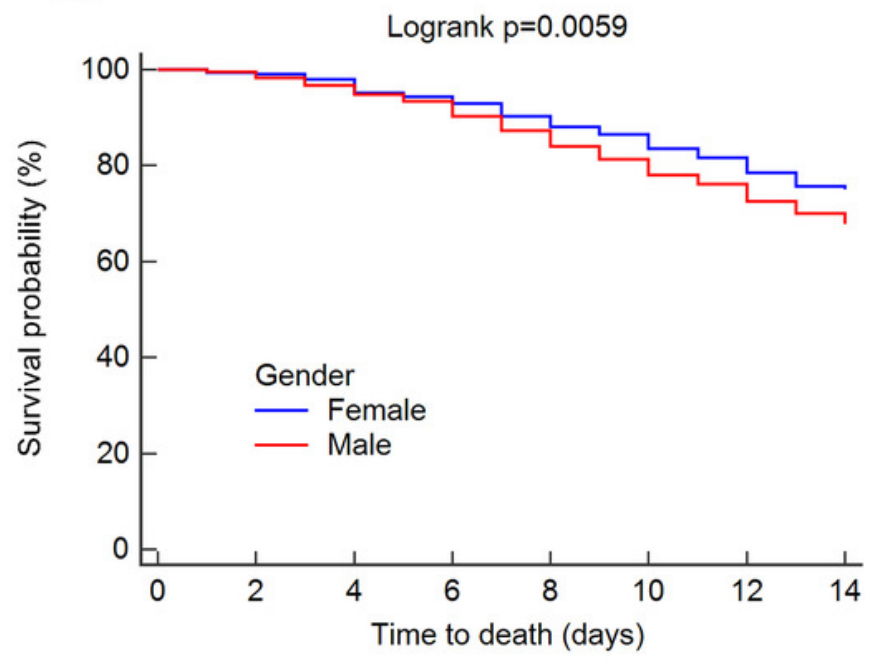

D

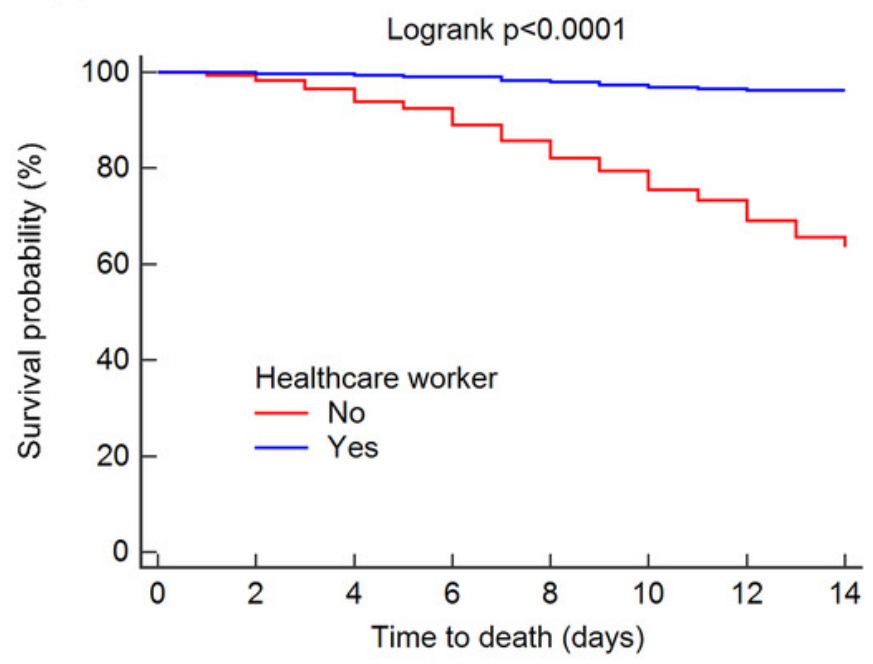

$\mathbf{F}$

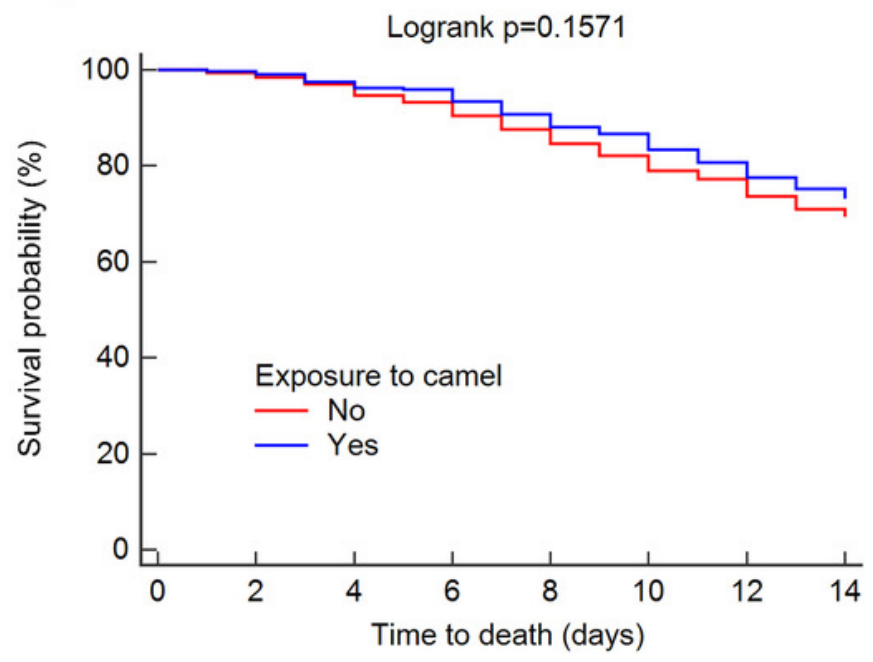




\section{Figure 4}

Figure 4

Survival rates for MERS-CoV for 45-day mortality at the Saudi national level between 2012 and 2019 by (A) region, (B) gender, (C) age group, (D) healthcare worker, (E) comorbidity, and $(F)$ exposure to camels 
A

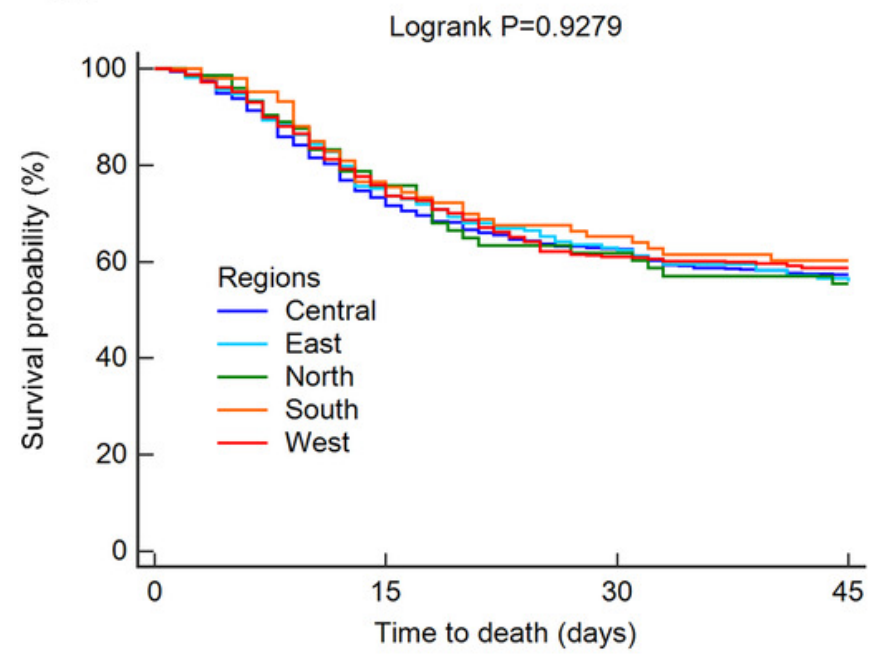

C

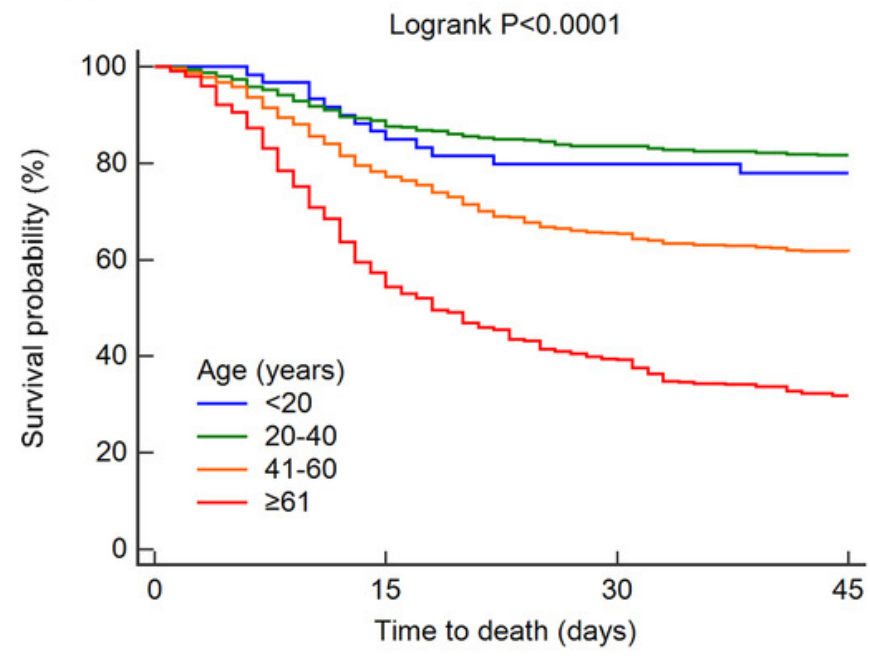

E

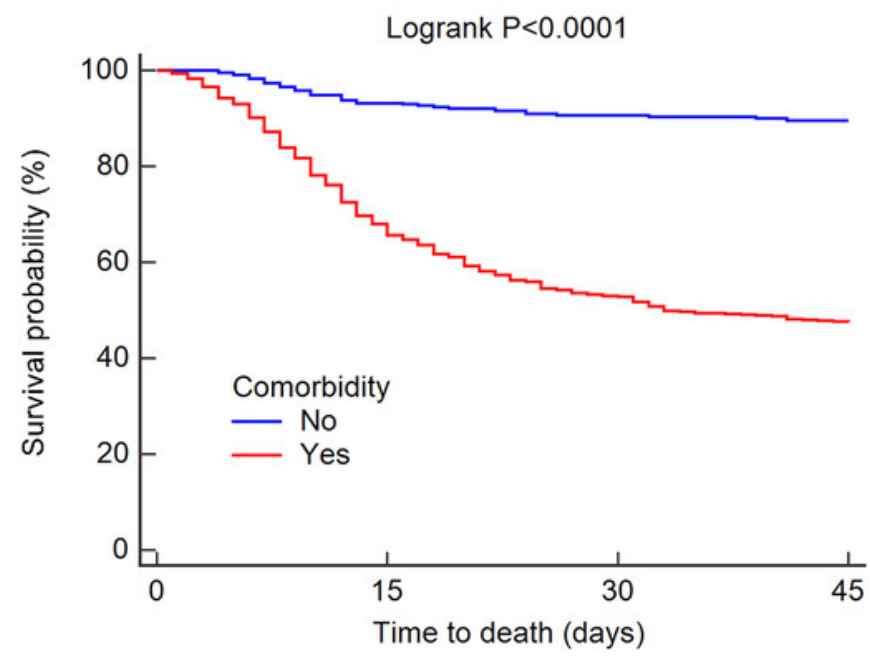

B

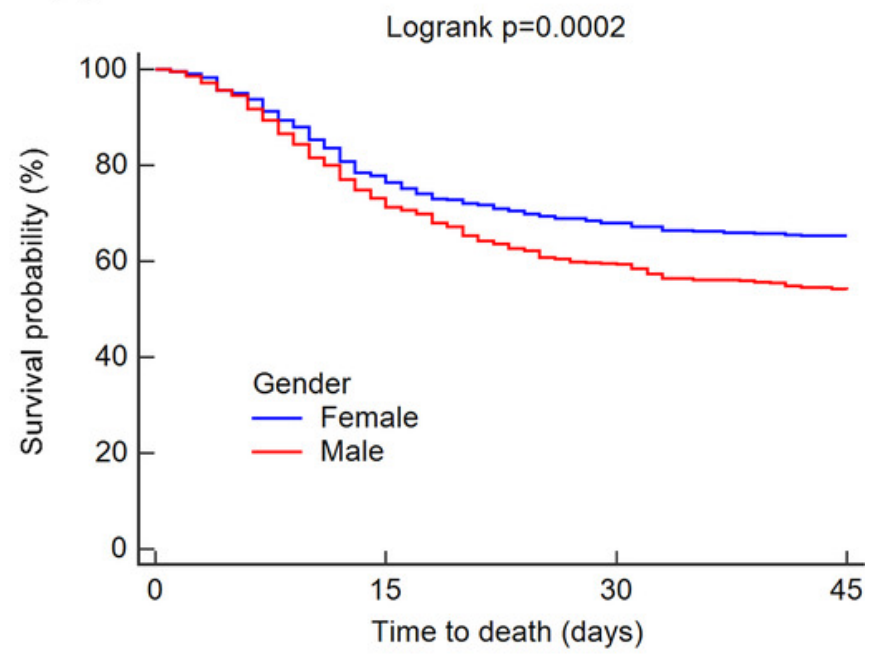

\section{D}

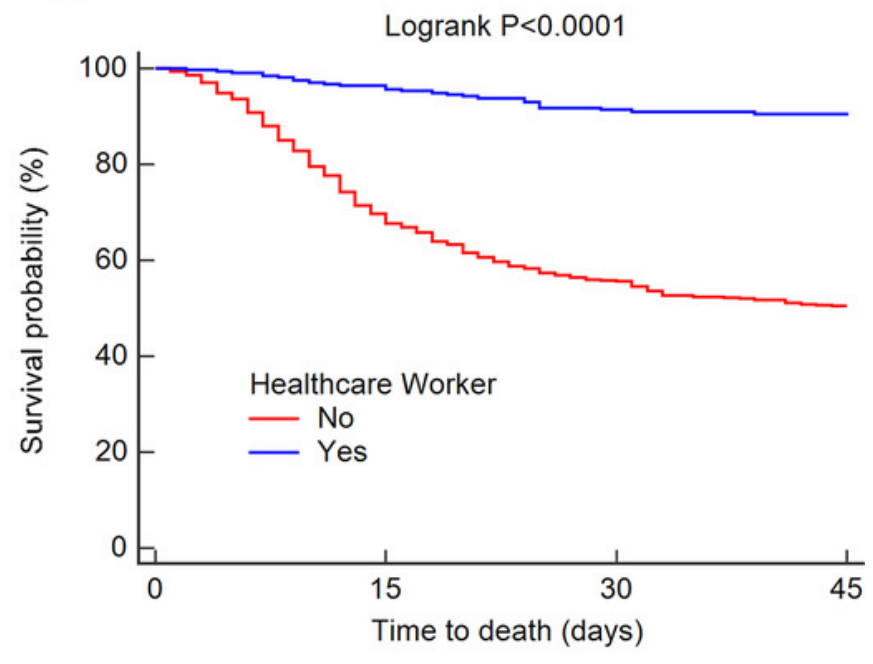

F

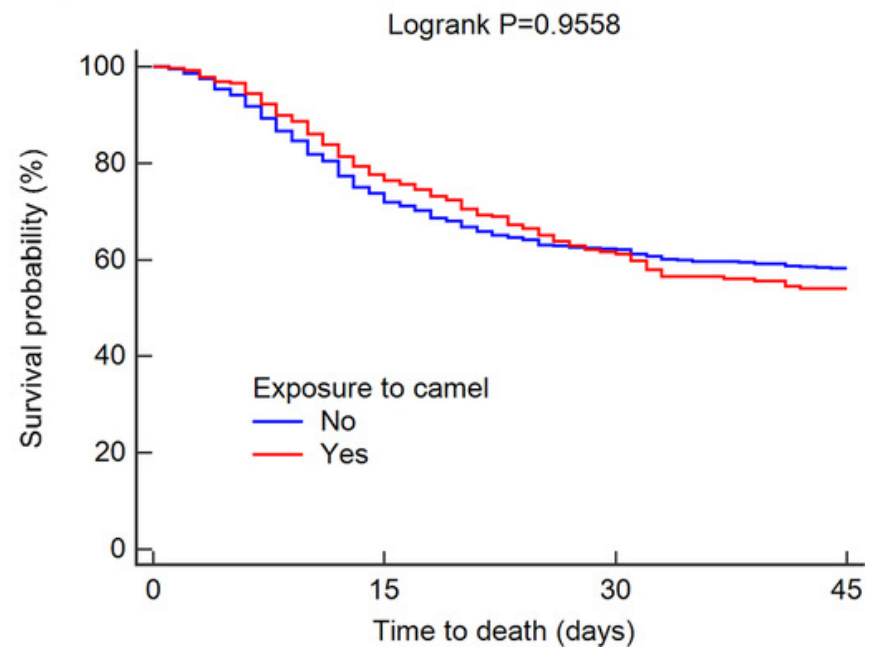


Figure 5

Figure 5

Survival rates for MERS-CoV for 14-day mortality at the Saudi regional level between 2012 and 2019 by gender, age group, healthcare worker, comorbidity, and exposure to camels for the (A-E) Central, (F-J) East, (K-O) West, (P-T) North, and (U-Y) South regions.
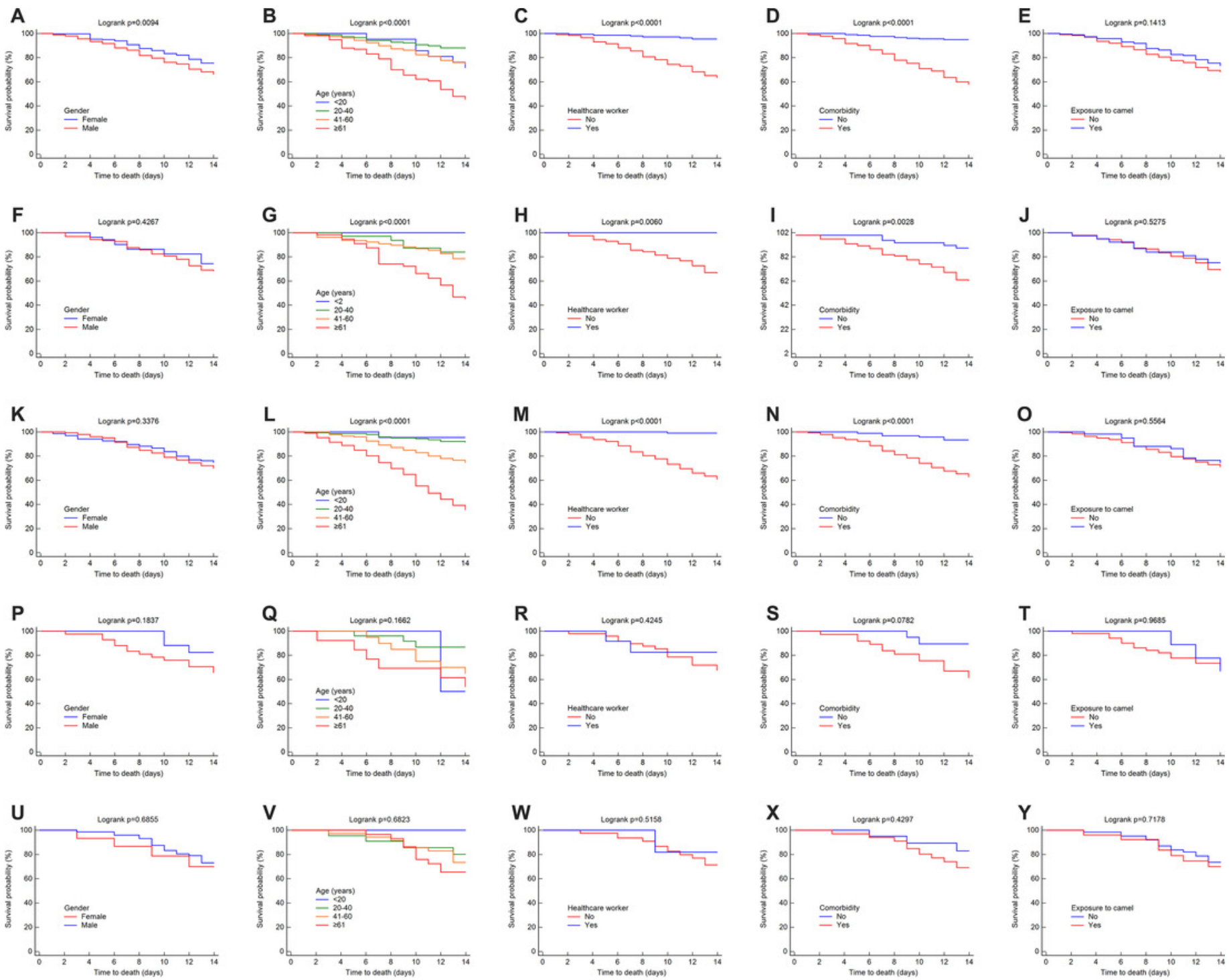


\section{Figure 6}

Figure 6

Spatial distribution of hazard ratios for MERS-CoV mortality risk factors based on multivariate analysis for each region between 2012 and 2019 for 45-day mortality by (A) age 41-60, (B) age $\geq 61$, (C) non-healthcare worker, and (D) comorbidity. For 45-day mortality by (E) age 41-60, (F) age $\geq 61,(G)$ non-healthcare worker, and $(H)$ comorbidity. 

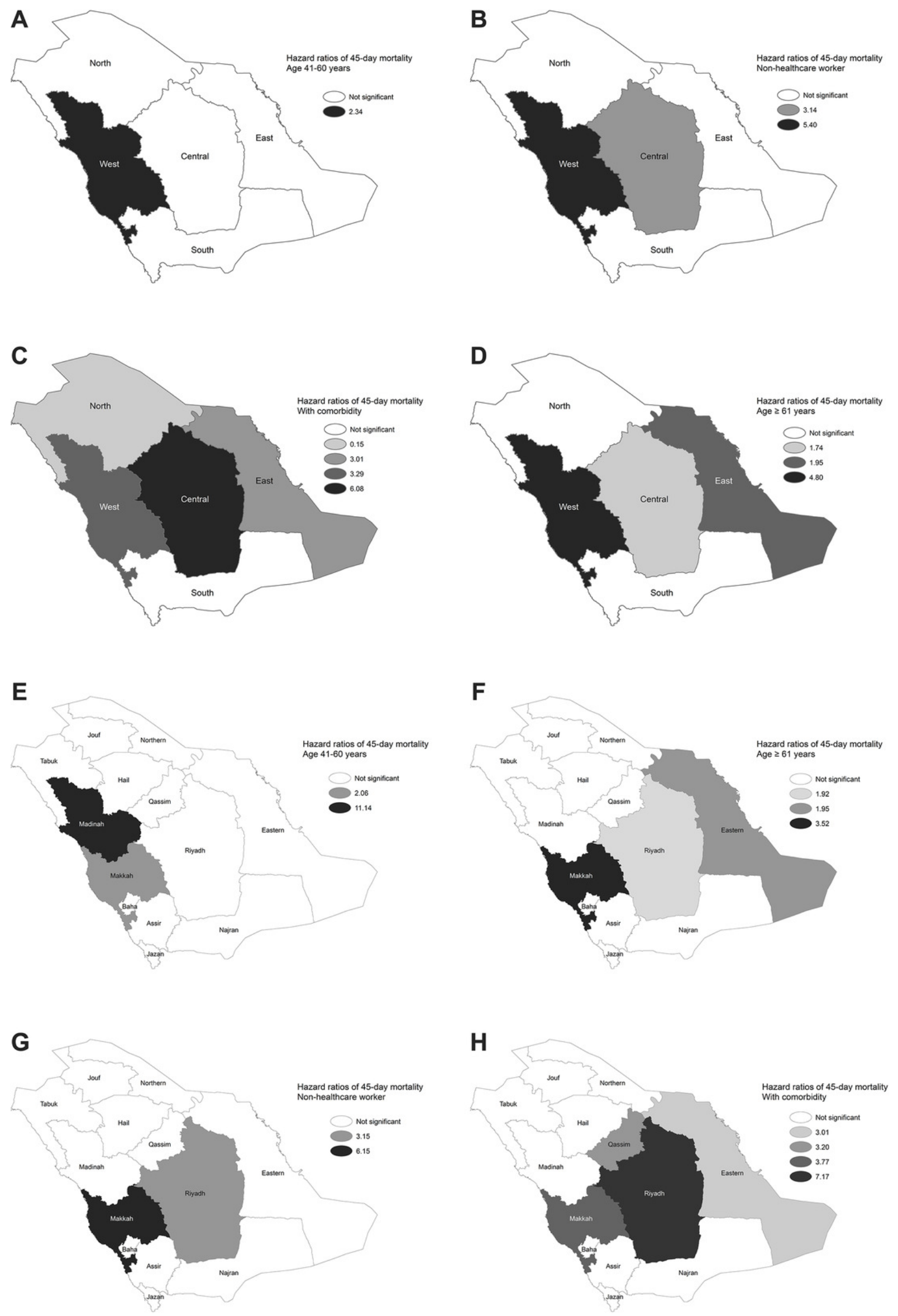

PeerJ reviewing PDF | (2020:01:44577:2:0:NEW 24 Jul 2020) 